\title{
The JCMT Transient Survey: Stochastic and Secular Variability of Protostars and Disks In the Submillimeter Region Observed over 18 Months
}

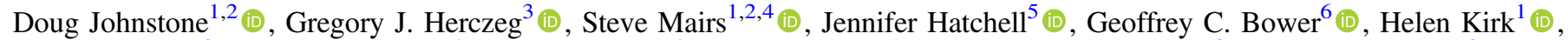 \\ James Lane $^{2}$ (i), Graham S. Bell ${ }^{4}$ (1), Sarah Graves ${ }^{4}$ (1), Yuri Aikawa ${ }^{7}$ (10), Huei-Ru Vivien Chen ${ }^{8}$ (10), Wen-Ping Chen ${ }^{9}$ (1),

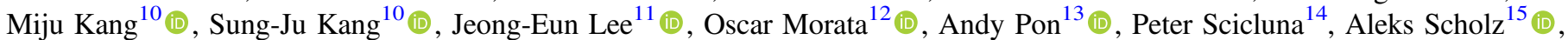 \\ Satoko Takahashi ${ }^{16,17}$, and Hyunju Yoo ${ }^{11,18}$ \\ The JCMT Transient Team \\ ${ }^{1}$ NRC Herzberg Astronomy and Astrophysics, 5071 West Saanich Road, Victoria, BC, V9E 2E7, Canada \\ ${ }^{2}$ Department of Physics and Astronomy, University of Victoria, Victoria, BC, V8P 5C2, Canada \\ ${ }^{3}$ Kavli Institute for Astronomy and Astrophysics, Peking University, Yiheyuan 5, Haidian Qu, 100871 Beijing, People's Republic of China \\ ${ }^{4}$ East Asian Observatory, 660 North A'ohōkū Place, University Park, Hilo, HI 96720, USA \\ ${ }^{5}$ Physics and Astronomy, Exeter University, Stocker Road, Exeter EX4 4QL, UK \\ ${ }^{6}$ Academia Sinica Institute of Astronomy and Astrophysics, 645 N. A'ohoku Place, Hilo, HI 96720, USA \\ ${ }^{7}$ Department of Astronomy, University of Tokyo, Tokyo, Japan \\ ${ }^{8}$ Department of Physics and Institute of Astronomy, National Tsing Hua University, Taiwan \\ ${ }^{9}$ Graduate Institute of Astronomy, National Central University, 300 Jhongda Road, Zhongli, Taoyuan, Taiwan \\ ${ }^{10}$ Korea Astronomy and Space Science Institute, 776 Daedeokdae-ro, Yuseong-gu, Daejeon 34055, Republic of Korea \\ ${ }^{11}$ School of Space Research, Kyung Hee University, 1732, Deogyeong-Daero, Giheung-gu Yongin-shi, Gyunggi-do 17104, Republic of Korea \\ ${ }^{12}$ Academia Sinica Institute of Astronomy and Astrophysics, 11F of AS/NTU Astronomy-Mathematics Building, No.1, Section 4 \\ Roosevelt Road, Taipei 10617, Taiwan
${ }^{13}$ Department of Physics and Astronomy, The University of Western Ontario, 1151 Richmond Street, London, ON, N6A 3K7, Canada \\ ${ }^{14}$ Academia Sinica Institute of Astronomy and Astrophysics, P.O. Box 23-141, Taipei 10617, Taiwan \\ ${ }^{15}$ SUPA, School of Physics \& Astronomy, North Haugh, St Andrews KY16 9SS, UK \\ ${ }^{16}$ Joint ALMA Observatory, Alonso de Córdova 3107, Vitacura, Santiago, Chile \\ ${ }^{17}$ National Astronomical Observatory of Japan, 2-21-1 Osawa, Mitaka, Tokyo 181-8588, Japan \\ ${ }^{18}$ Department of Astronomy and Space Science, Chungnam National University, 99 Daehak-ro, Yuseong-gu, Daejeon 34134, Republic of Korea \\ Received 2017 October 30; revised 2018 January 4; accepted 2018 January 10; published 2018 February 8
}

\begin{abstract}
We analyze results from the first 18 months of monthly submillimeter monitoring of eight star-forming regions in the JCMT Transient Survey. In our search for stochastic variability in 1643 bright peaks, only the previously identified source, EC 53, shows behavior well above the expected measurement uncertainty. Another four sources-two disks and two protostars-show moderately enhanced standard deviations in brightness, as expected for stochastic variables. For the two protostars, this apparent variability is the result of single epochs that are much brighter than the mean. In our search for secular brightness variations that are linear in time, we measure the fractional brightness change per year for 150 bright peaks, 50 of which are protostellar. The ensemble distribution of slopes is well fit by a normal distribution with $\sigma \sim 0.023$. Most sources are not rapidly brightening or fading at submillimeter wavelengths. Comparison against time-randomized realizations shows that the width of the distribution is dominated by the uncertainty in the individual brightness measurements of the sources. A toy model for secular variability reveals that an underlying Gaussian distribution of linear fractional brightness change $\sigma=0.005$ would be unobservable in the present sample, whereas an underlying distribution with $\sigma=0.02$ is ruled out. Five protostellar sources, $10 \%$ of the protostellar sample, are found to have robust secular measures deviating from a constant flux. The sensitivity to secular brightness variations will improve significantly with a sample over a longer time duration, with an improvement by factor of two expected by the conclusion of our 36 month survey.
\end{abstract}

Key words: galaxies: star formation - stars: protostars

\section{Introduction}

A protostellar core begins its life by growing smoothly from a collapsing envelope. Protoplanetary disks are thought to form early in the protostellar lifetime (e.g., Jørgensen et al. 2008); once formed, the disk channels accretion from the envelope onto the star (e.g., Hartmann et al. 1997). While the initial protostellar growth from the envelope should be steady, disk accretion is expected to be variable because instabilities are expected to grow quickly (see review by Armitage 2015).

These instabilities produce macroscopic structures that are detectable even in young disks, such as rings in $\mathrm{HL}$ Tau (ALMA Partnership et al. 2015), spiral density waves in Elias 2-27 (Pérez et al. 2016), and multiplicity from disk fragmentation in L1448 IRS3B (Tobin et al. 2016a). However, while such structures are detectable at large radii, the identification of structures in inner disks is typically beyond the detection limits of current instrumentation. The size and scale of instabilities in the inner disk may instead be indirectly traced by monitoring accretion from the disk onto the star. Variability in accretion is commonly detected in optically visible systems and has been used to infer the presence of instabilities in the disk (see reviews by Audard et al. 2014; Hartmann et al. 2016). Small, short-lived flickers seen in high-cadence monitoring (e.g., Cody et al. 2017) and spectroscopic campaigns (e.g., Costigan et al. 2014) suggest a non-steady star-disk connection (e.g., Romanova et al. 2012). Months-long bursts of EXor systems 
may reveal the expansion and contraction of the magnetospheric cavity (D'Angelo \& Spruit 2010). The largest known bursts, seen as FUor objects, are caused by an increase by a factor of $10^{4}$ in the accretion rate and may last for over a century (e.g., Zhu et al. 2009). Recently, Liu et al. (2017) surveyed a sample of 29 FUors and Exors at $1.3 \mathrm{~mm}$ with the Submillimeter Array and tentatively detected two sources, V2494 Cyg and V2495 Cyg, with 30\%-60\% variability in the millimeter flux on timescales of a year.

This variability in accretion, and large bursts in particular, is thought to play an important role in the chemical evolution of the envelope and disk (e.g., Kim et al. 2012; Harsono et al. 2015; Frimann et al. 2017) as well as the contraction rate of the star (e.g., Hosokawa et al. 2011; Baraffe et al. 2017). However, at younger stages of evolution, the star-disk system is obscured by an optically thick envelope that enshrouds the accretion, preventing direct detection of accretion and therefore any variability in it. Yet during this period, the star accretes most of its mass, and the effects of any variability are expected to be the most significant.

Johnstone et al. (2013) examined the variability of submillimeter dust continuum emission as a new method to probe the variability of accretion onto the star. They noted that the variable mass accretion $\dot{M}_{a}(t)$ with time, $t$, onto deeply embedded protostars should be observable through the proxy measurement of accretion luminosity, $L_{a}(t) \propto \dot{M}_{a}(t)$, and that this varying accretion luminosity should leave a signature on the protostellar envelope. Since the heat capacity of dust is small, the absorption and re-emission from dust within the dense envelope should quickly bring the spectral energy distribution (SED) of the protostellar core into an equilibrium determined by the new accretion luminosity. Indeed, the only relevant time delay was found to be the light-crossing time, of the order of days to months depending on the size of the envelope and the wavelength of interest. The longest delay times are associated with single-dish submillimeter observations, since for these observations the change in the temperature of the outer envelope is responsible for the majority of the observed change in emission. The change in the temperature of the gas proceeds much more slowly than for the dust, because the gas has a much higher heat capacity and is warmed (or cooled) primarily through collisions with the dust.

To search for variability in submillimeter dust emission, "The JCMT Transient Survey" (Herczeg et al. 2017) is monitoring eight star-forming regions with the SCUBA-2 (Holland et al. 2013) submillimeter bolometer on the James Clerk Maxwell Telescope (JCMT). These star-forming regions are imaged with an approximately monthly cadence in order to search for indicators of variability. Our survey was begun in 2015 December and will run through at least 2019 January. Each region has a sufficient number of compact bright submillimeter sources to allow for significant improvement in the nominal pointing and brightness calibration (Mairs et al. 2017a). This survey has already uncovered the first robust detection of a submillimeter periodic variable (EC 53, Yoo et al. 2017). Through comparison with previous observations by the Gould Belt Survey (GBS; Ward-Thompson et al. 2007) of these same star-forming regions, it has also revealed that a handful of sources have small but robust variations in their brightness across two to four years (Mairs et al. 2017b).

In this paper we analyze the first 18 months of the JCMT Transient Survey to search for evidence of stochastic and secular submillimeter variability within eight star-forming regions. In Section 2 we recap the data reduction and calibration procedures that are applied to the individual observations to ensure a reliable and uniform data product. Using deep, stacked images of each region we determine the location of robust submillimeter peaks and collate these sources with known protostars and disk sources. In Section 3 we analyze, for each identified source, the standard deviation in the brightness across all epochs and search for those sources that show significant variation from the expected value, a potential signpost of stochastic variable behavior. In Section 4 we measure the degree of secular variability in the brightness observed for each source by fitting linear slopes in time to the brightness measurements. Consideration of the uncertainty in the slope measurement and comparison against timerandomized observations of the same sources allow for the determination of robust results. In Section 5 we discuss individual sources in more detail, looking at both the robust and candidate sources uncovered in the previous two sections as well as potential variable sources obtained from the literature. Section 6 places the observations obtained to date with the JCMT Transient Survey in context with the broader search for variability in both observations and numerical simulations. Finally, in Section 7 we summarize our results.

\section{Data Reduction}

The observations included in this paper were taken as part of the JCMT Transient Survey (Herczeg et al. 2017), with roughly monthly observations from 2015 December 22 through 2017 June 16. The JCMT Transient Survey uses the Submillimetre Common User Bolometer Array 2 (SCUBA-2; Holland et al. 2013) to simultaneously image the sky at both $850 \mu \mathrm{m}$ and $450 \mu \mathrm{m}$ with effective beam FWHMs of $14 . " 6$ and 9!" 8 , respectively. In this paper, we focus only on the $850 \mu \mathrm{m}$ images. The $450 \mu \mathrm{m}$ data are far more susceptible to slight changes in the precipitable water vapour, and this introduces complications to the data reduction and calibration procedures for a consistent comparison of peak brightnesses across many epochs. All of the observations were performed while the optical depth at $225 \mathrm{GHz}$ $\left(\tau_{225}\right)$ was less than 0.12 as measured by the JCMT water vapour radiometer (Dempsey \& Friberg 2008). The integration time of the observations varied between 20 and 40 minutes, depending on weather conditions, in order to achieve a consistent sensitivity of $\sim 10 \mathrm{mJy} \mathrm{beam}^{-1}$ at $850 \mu \mathrm{m}$. The $850 \mu \mathrm{m}$ data were reduced and calibrated following the procedure described by Mairs et al. (2017a) using the iterative map-making software MAKEMAP (described in detail by Chapin et al. 2013) in the SMURF package (Jenness et al. 2013) found within the STARLINK software (Currie et al. 2014). Each $850 \mu \mathrm{m}$ map is gridded to $3^{\prime \prime}$ pixels and convergence of the iterative solution is defined when the difference in individual pixels changes on average by $<0.1 \%$ of the rms noise present in the map. Emission on scales larger than $\sim 200^{\prime \prime}$ is filtered out of these maps while smaller-scale structures are robustly recovered (see Chapin et al. 2013; Mairs et al. 2015, 2017a). While the $\operatorname{CO}(J=3-2)$ emission line contributes to the flux measured in $850 \mu \mathrm{m}$ continuum observations (Johnstone \& Bally 1999; Drabek et al. 2012; Coudé et al. 2016), Mairs et al. (2016) show that the peak brightnesses of compact sources are not significantly affected by the removal of this line, and therefore no attempt to remove the line has been undertaken for this analysis.

The pertinent observation and calibration information is provided in Table 1. The Transient Survey calibration procedure (Mairs et al. 2017a) improves on the default JCMT calibrations 
Table 1

Regions, Epochs, and Calibration

\begin{tabular}{|c|c|c|c|c|c|c|c|c|}
\hline Region & R.A. & Decl. & Epoch & Date & Scan & $\tau_{225}{ }^{\mathrm{a}}$ & $\begin{array}{c}\text { Noise } \\
\left(\text { Jy beam }^{-1}\right)\end{array}$ & $\mathrm{FCF}^{\mathrm{b}}$ \\
\hline \multirow[t]{10}{*}{ IC 348} & $3^{\mathrm{h}} 44^{\mathrm{m}} 18^{\mathrm{s}} .00$ & $+32: 04: 59.00$ & 1 & 2015 Dec 22 & 00019 & 0.064 & 0.011 & 1.022 \\
\hline & & & 2 & 2016 Jan 15 & 00022 & 0.072 & 0.009 & 1.050 \\
\hline & & & 3 & 2016 Feb 05 & 00018 & 0.037 & 0.013 & 0.968 \\
\hline & & & 4 & 2016 Feb 26 & 00020 & 0.054 & 0.012 & 0.973 \\
\hline & & & 5 & 2016 Mar 18 & 00027 & 0.048 & 0.011 & 0.942 \\
\hline & & & 6 & 2016 Apr 17 & 00009 & 0.036 & 0.011 & 0.927 \\
\hline & & & 7 & 2016 Aug 26 & 00040 & 0.082 & 0.014 & 0.968 \\
\hline & & & 8 & 2016 Nov 26 & 00022 & 0.049 & 0.010 & 1.226 \\
\hline & & & 9 & 2017 Feb 09 & 00028 & 0.089 & 0.012 & 1.030 \\
\hline & & & 10 & 2017 Mar 20 & 00019 & 0.086 & 0.011 & 0.980 \\
\hline \multirow[t]{11}{*}{ NGC 1333} & $3^{\mathrm{h}} 28^{\mathrm{m}} 54^{\mathrm{s}} .00$ & $+31: 16: 52.00$ & 1 & 2015 Dec 22 & 00018 & 0.061 & 0.011 & 1.039 \\
\hline & & & 2 & 2016 Jan 15 & 00010 & 0.081 & 0.012 & 0.978 \\
\hline & & & 3 & 2016 Feb 05 & 00017 & 0.037 & 0.012 & 1.001 \\
\hline & & & 4 & 2016 Feb 29 & 00017 & 0.042 & 0.011 & 1.001 \\
\hline & & & 5 & 2016 Mar 25 & 00011 & 0.057 & 0.011 & 0.927 \\
\hline & & & 6 & 2016 Aug 02 & 00031 & 0.094 & 0.012 & 1.027 \\
\hline & & & 7 & 2016 Aug 30 & 00048 & 0.090 & 0.013 & 1.020 \\
\hline & & & 8 & 2016 Nov 19 & 00088 & 0.067 & 0.008 & 1.058 \\
\hline & & & 9 & 2016 Nov 26 & 00021 & 0.048 & 0.010 & 1.164 \\
\hline & & & 10 & 2017 Feb 06 & 00029 & 0.124 & 0.013 & 0.948 \\
\hline & & & 11 & 2017 Mar 18 & 00015 & 0.111 & 0.014 & 0.948 \\
\hline \multirow[t]{13}{*}{ NGC 2024} & $5^{\mathrm{h}} 41^{\mathrm{m}} 41^{\mathrm{s}} .00$ & $-01: 53: 51.00$ & 1 & 2015 Dec 26 & 00049 & 0.117 & 0.013 & 0.975 \\
\hline & & & 2 & 2016 Jan 16 & 00022 & 0.057 & 0.009 & 0.999 \\
\hline & & & 3 & 2016 Feb 06 & 00013 & 0.043 & 0.011 & 1.046 \\
\hline & & & 4 & 2016 Feb 29 & 00022 & 0.044 & 0.011 & 1.089 \\
\hline & & & 5 & 2016 Mar 25 & 00021 & 0.057 & 0.012 & 0.935 \\
\hline & & & 6 & 2016 Mar 29 & 00010 & 0.053 & 0.008 & 1.019 \\
\hline & & & 7 & 2016 Apr 27 & 00012 & 0.052 & 0.013 & 0.843 \\
\hline & & & 8 & 2016 Aug 26 & 00029 & 0.092 & 0.012 & 1.001 \\
\hline & & & 9 & 2016 Nov 19 & 00099 & 0.067 & 0.009 & 1.010 \\
\hline & & & 10 & 2016 Nov 26 & 00053 & 0.063 & 0.008 & 1.157 \\
\hline & & & 11 & 2017 Feb 06 & 00025 & 0.111 & 0.011 & 1.054 \\
\hline & & & 12 & 2017 Mar 19 & 00010 & 0.103 & 0.011 & 0.983 \\
\hline & & & 13 & 2017 Apr 23 & 00011 & 0.077 & 0.011 & 0.941 \\
\hline \multirow[t]{12}{*}{ NGC 2068} & $5^{\mathrm{h}} 46^{\mathrm{m}} 13^{\mathrm{s}} \cdot 00$ & $+00: 06: 05.00$ & 1 & 2015 Dec 26 & 00052 & 0.116 & 0.013 & 0.957 \\
\hline & & & 2 & 2016 Jan 16 & 00027 & 0.058 & 0.008 & 1.145 \\
\hline & & & 3 & 2016 Feb 06 & 00015 & 0.046 & 0.011 & 1.086 \\
\hline & & & 4 & 2016 Feb 29 & 00013 & 0.042 & 0.012 & 0.991 \\
\hline & & & 5 & 2016 Mar 29 & 00011 & 0.056 & 0.010 & 1.010 \\
\hline & & & 6 & 2016 Apr 27 & 00013 & 0.052 & 0.016 & 0.774 \\
\hline & & & 7 & 2016 Aug 27 & 00053 & 0.083 & 0.012 & 0.966 \\
\hline & & & 8 & 2016 Nov 20 & 00088 & 0.093 & 0.012 & 0.935 \\
\hline & & & 9 & 2016 Nov 26 & 00056 & 0.063 & 0.009 & 1.132 \\
\hline & & & 10 & 2017 Feb 06 & 00017 & 0.113 & 0.012 & 1.045 \\
\hline & & & 11 & 2017 Mar 19 & 00014 & 0.112 & 0.012 & 1.020 \\
\hline & & & 12 & 2017 Apr 21 & 00025 & 0.091 & 0.015 & 0.846 \\
\hline \multirow[t]{11}{*}{ OMC $2 / 3$} & $5^{\mathrm{h}} 35^{\mathrm{m}} 31^{\mathrm{s}} .00$ & $-05: 00: 38.00$ & 1 & 2015 Dec 26 & 00036 & 0.109 & 0.011 & 1.039 \\
\hline & & & 2 & 2016 Jan 16 & 00019 & 0.057 & 0.009 & 1.013 \\
\hline & & & 3 & 2016 Feb 06 & 00012 & 0.041 & 0.011 & 1.023 \\
\hline & & & 4 & 2016 Feb 29 & 00011 & 0.043 & 0.012 & 0.917 \\
\hline & & & 5 & 2016 Mar 25 & 00015 & 0.056 & 0.011 & 0.974 \\
\hline & & & 6 & 2016 Apr 22 & 00011 & 0.050 & 0.011 & 0.966 \\
\hline & & & 7 & 2016 Aug 26 & 00020 & 0.106 & 0.014 & 1.016 \\
\hline & & & 8 & 2016 Nov 26 & 00052 & 0.062 & 0.008 & 1.125 \\
\hline & & & 9 & 2017 Feb 06 & 00021 & 0.115 & 0.011 & 1.032 \\
\hline & & & 10 & 2017 Mar 18 & 00012 & 0.102 & 0.011 & 0.959 \\
\hline & & & 11 & 2017 Apr 21 & 00022 & 0.090 & 0.014 & 0.865 \\
\hline & $16^{\mathrm{h}} 27^{\mathrm{m}} 05^{\mathrm{s}} .00$ & $-24: 32: 37.00$ & 1 & 2016 Jan 15 & 00084 & 0.067 & 0.012 & 0.913 \\
\hline \multirow{2}{*}{$\begin{array}{l}\text { Oph } \\
\text { Core }\end{array}$} & & & 2 & 2016 Feb 05 & 00063 & 0.038 & 0.011 & 1.100 \\
\hline & & & 3 & 2016 Feb 26 & 00051 & 0.045 & 0.010 & 1.095 \\
\hline
\end{tabular}


Table 1

(Continued)

\begin{tabular}{|c|c|c|c|c|c|c|c|c|}
\hline Region & R.A. & Decl. & Epoch & Date & Scan & $\tau_{225}{ }^{\mathrm{a}}$ & $\begin{array}{c}\text { Noise } \\
\left(\mathrm{Jy} \mathrm{beam}^{-1}\right)\end{array}$ & $\mathrm{FCF}^{\mathrm{b}}$ \\
\hline & & & 4 & 2016 Mar 19 & 00065 & 0.044 & 0.011 & 1.073 \\
\hline & & & 5 & 2016 Apr 17 & 00043 & 0.038 & 0.011 & 1.048 \\
\hline & & & 6 & 2016 May 21 & 00034 & 0.076 & 0.016 & 0.933 \\
\hline & & & 7 & 2016 Aug 26 & 00011 & 0.108 & 0.018 & 0.935 \\
\hline & & & 8 & 2017 Feb 06 & 00083 & 0.110 & 0.013 & 0.998 \\
\hline & & & 9 & 2017 Mar 20 & 00053 & 0.062 & 0.010 & 1.042 \\
\hline & & & 10 & 2017 Apr 19 & 00033 & 0.105 & 0.015 & 1.003 \\
\hline & & & 11 & 2017 May 18 & 00028 & 0.039 & 0.011 & 1.119 \\
\hline & & & 12 & 2017 Jun 16 & 00010 & 0.124 & 0.013 & 1.028 \\
\hline \multirow{16}{*}{$\begin{array}{l}\text { Serpens } \\
\text { Main }\end{array}$} & $18^{\mathrm{h}} 29^{\mathrm{m}} 49^{\mathrm{s}} .00$ & $+01: 15: 20.00$ & 1 & 2016 Feb 02 & 00054 & 0.091 & 0.012 & 0.972 \\
\hline & & & 2 & 2016 Feb 23 & 00050 & 0.050 & 0.011 & 1.032 \\
\hline & & & 3 & 2016 Mar 17 & 00051 & 0.040 & 0.012 & 0.954 \\
\hline & & & 4 & 2016 Apr 15 & 00046 & 0.040 & 0.010 & 1.118 \\
\hline & & & 5 & 2016 May 21 & 00039 & 0.077 & 0.014 & 0.988 \\
\hline & & & 6 & 2016 Jul 22 & 00023 & 0.096 & 0.012 & 0.989 \\
\hline & & & 7 & 2016 Aug 27 & 00012 & 0.087 & 0.011 & 1.012 \\
\hline & & & 8 & 2016 Sep 29 & 00012 & 0.094 & 0.012 & 0.963 \\
\hline & & & 9 & $2017 \mathrm{Feb} 22$ & 00070 & 0.097 & 0.010 & 1.077 \\
\hline & & & 10 & 2017 Mar 20 & 00056 & 0.066 & 0.010 & 1.029 \\
\hline & & & 11 & 2017 Apr 03 & 00053 & 0.063 & 0.010 & 1.041 \\
\hline & & & 12 & 2017 Apr 17 & 00044 & 0.057 & 0.009 & 1.133 \\
\hline & & & 13 & 2017 May 05 & 00035 & 0.046 & 0.008 & 1.179 \\
\hline & & & 14 & 2017 May 19 & 00030 & 0.105 & 0.012 & 1.049 \\
\hline & & & 15 & 2017 Jun 02 & 00041 & 0.079 & 0.010 & 1.086 \\
\hline & & & 16 & 2017 Jun 16 & 00025 & 0.100 & 0.010 & 1.027 \\
\hline \multirow{13}{*}{$\begin{array}{l}\text { Serpens } \\
\text { South }\end{array}$} & $18^{\mathrm{h}} 29^{\mathrm{m}} 62^{\mathrm{s}} .00$ & $-02: 02: 48.00$ & 1 & 2016 Feb 02 & 00058 & 0.093 & 0.011 & 0.974 \\
\hline & & & 2 & 2016 Feb 23 & 00065 & 0.053 & 0.010 & 1.054 \\
\hline & & & 3 & 2016 Mar 17 & 00052 & 0.042 & 0.013 & 0.911 \\
\hline & & & 4 & 2016 Apr 15 & 00048 & 0.040 & 0.010 & 1.085 \\
\hline & & & 5 & 2016 May 21 & 00044 & 0.074 & 0.013 & 0.983 \\
\hline & & & 6 & $2016 \mathrm{Jul} 21$ & 00011 & 0.077 & 0.012 & 1.024 \\
\hline & & & 7 & 2016 Aug 27 & 00017 & 0.092 & 0.012 & 0.979 \\
\hline & & & 8 & 2016 Sep 29 & 00018 & 0.083 & 0.012 & 0.931 \\
\hline & & & 9 & 2017 Feb 22 & 00081 & 0.099 & 0.009 & 1.165 \\
\hline & & & 10 & 2017 Mar 22 & 00094 & 0.068 & 0.010 & 1.085 \\
\hline & & & 11 & 2017 Apr 19 & 00036 & 0.109 & 0.015 & 1.028 \\
\hline & & & 12 & 2017 May 18 & 00037 & 0.042 & 0.010 & 1.098 \\
\hline & & & 13 & 2017 Jun 16 & 00021 & 0.104 & 0.011 & 1.007 \\
\hline
\end{tabular}

Notes.

a The optical depth at $225 \mathrm{GHz}$.

$\mathrm{b}$ The flux calibration shown is the factor applied to the epoch after data reduction using the observatory-defined default calibration factor.

(Dempsey et al. 2013) in two ways. First, image alignment is improved from the $2^{\prime \prime}-6^{\prime \prime}$ uncertainty in telescope pointing to a relative value per field of $<1^{\prime \prime}$. Second, the default brightness calibration of $\sim 5 \%-10 \%$ is lowered to a relative value per field per epoch of $\sim 2 \%$ (for details refer to Mairs et al. 2017a).

For each of the eight observed regions, the individual calibrated epochs were stacked to produce a deep mean image. The source finding routine JSA_Catalogue (found in Starlink's PICARD package, Gibb et al. 2013) was then applied to search for locations of peak brightness. JSA_Catalogue optimizes the user inputs of the FellWalker algorithm (Berry 2015) to identify compact, peaked emission and the associated, larger-scale structure. In this work, only the former is considered. Identified peaks must have a brightness at least five times the locally derived rms noise and an area of at least 81 square arcseconds ( 9 pixels). These pixels must be adjacent and the peak width in both the horizontal and vertical dimensions must be at least 2 pixels. Nearby peaks are merged into a single object if the flux between them does not drop by a level of at least five times the rms noise.

The resulting 1643 submillimeter peaks were then collated against master catalogs of disks (Class II) and protostars (Class 0/I) identified by the Spitzer Space Telescope (Megeath et al. 2012; Dunham et al. 2015) and the Herschel Space Observatory (Stutz et al. 2013), with matches assumed for sources separated by less than $10^{\prime \prime}$ (for details see Mairs et al. 2017a). ${ }^{19}$ Table 2 provides statistics on the numbers of

\footnotetext{
19 One source in NGC 2068 was originally classified as starless since it is not found in either the Spitzer catalog of Orion young stellar objects (Megeath et al. 2012) or the Herschel supplementary list of new protostars (Stutz et al. 2013). After being identified as a robust secular variable (see Section 4 below), a literature search uncovered it as a PACS Bright Red Source (PBRS) identified by Stutz et al. (2013). PBRS properties are consistent with those of young Class 0 protostars. The source is known as HOPS 373 (see also Furlan et al. 2016).
} 
Table 2

Number of Peaks Extracted from Co-added Images

\begin{tabular}{|c|c|c|c|c|c|c|}
\hline Region & All Peaks & $\begin{array}{c}\text { Peaks } \\
\left(>0.35 \quad \text { Jy beam }^{-1}\right)\end{array}$ & All Protostars & $\begin{array}{c}\text { Protostars } \\
\left(>0.35 \quad \mathrm{Jy} \mathrm{beam}^{-1}\right)\end{array}$ & All Disks & $\begin{array}{c}\text { Disks } \\
\left(>0.35 \quad \text { Jy beam }^{-1}\right)\end{array}$ \\
\hline IC 348 & 79 & 3 & 6 & 3 & 5 & 0 \\
\hline NGC 1333 & 142 & 18 & 24 & 11 & 8 & 0 \\
\hline NGC 2024 & 369 & 11 & 5 & 2 & 15 & 1 \\
\hline NGC 2068 & 126 & 19 & 15 & 10 & 6 & 0 \\
\hline Oph Core & 257 & 14 & 24 & 2 & 24 & 4 \\
\hline OMC $2 / 3$ & 276 & 54 & 15 & 13 & 40 & 9 \\
\hline Serpens Main & 92 & 12 & 8 & 7 & 5 & 0 \\
\hline Serpens South & 302 & 20 & 18 & 3 & 8 & 0 \\
\hline Total & 1643 & 151 & 115 & 51 & 111 & 14 \\
\hline
\end{tabular}

sources of various types identified in each region as a function of limiting peak brightness. In the table we separate out the 151 sources brighter than $0.35 \mathrm{Jy}$ beam $^{-1}$, for which the fractional uncertainty in the individual measurements is less than $5 \%$ (see Equation (1) in Section 3).

The source identification algorithm used in this paper is significantly different than that used by Mairs et al. (2017a) to calibrate individual epochs. Mairs et al. use Gaussclumps (Stutzki \& Guesten 1990) to fit Gaussian profiles to peaks in individual epochs regions and then group Gaussians that share common coordinates across epochs. By fitting Gaussians, Mairs et al. (2017a) are able to centroid sources in images that are not yet spatially aligned, a key component of their calibration analysis. In the present analysis we identify the peak brightness location in the stacked mean map and then use this fixed location to compare across individual epochs, making explicit use of the calibration and alignment achieved by Mairs et al. (2017a).

\section{Stochasticity: Standard Deviation Analysis}

The large number of sources (greater than 1500 overall and more than 150 with peak brightness larger than $0.35 \mathrm{Jy}$ beam $^{-1}$ ), the multiple epochs (typically 12 per region), and the consistent observing conditions and calibration procedures provide a rich, uniform data set to inspect. We therefore search for evidence of variability, either stochastic or secular, via statistical investigations.

The first analysis that we perform is to measure the standard deviation of the brightness of each individual source, across all eight regions. For each source, $i$, the mean peak brightness in the stacked image, $f_{m}(i)$, is compared against the brightness at the same location in each individual epoch $n, f_{n}(i)$. The source variance, across all epochs, is then $v(i)=\sum\left[f_{m}(i)-f_{n}(i)\right]^{2}$ and the standard deviation is $\operatorname{SD}(i)=\left[v(i) /\left(n_{e}-1\right)\right]^{1 / 2}$ where $n_{e}$ is the number of epochs for which the region has been observed. The measurements of standard deviation in peak brightness for all sources are plotted against mean peak brightness in Figure 1, with colors denoting whether the source is associated with a known protostar (Class 0/I: red star) or a disk source (Class II: blue square).

We expect that the uncertainty in the brightness of faint sources will be dominated by the relatively uniform noise in a given epoch, $\Delta f$ (faint) $\sim 0.014$ Jy beam $^{-1}$, while for bright sources the uncertainty in the relative calibration of the map will dominate, $\Delta f$ (bright) $\sim 0.02 f$ (bright) (see Mairs et al. 2017b). Thus, we present a fiducial standard deviation model,
$\mathrm{SD}_{\text {fid }}$, where

$$
\operatorname{SD}_{\text {fid }}(i)=\left[(0.014)^{2}+\left(0.02 \times f_{m}(i)\right)^{2}\right]^{1 / 2} \mathrm{Jy} \mathrm{beam}^{-1} \text {. }
$$

The majority of the standard deviation measures in Figure 1 lie near the fiducial model, with some scatter. To show this result more clearly, Figure 2 plots the standard deviation in units of the fiducial model as a function of source brightness. Only one source stands out in this plot. EC 53, a protostar in Serpens Main with a peak flux of $1.15 \mathrm{Jy} \mathrm{beam}^{-1}$, was already identified as a submillimeter variable in our survey (Yoo et al. 2017), and was previously known as a Class I periodic variable at $2 \mu \mathrm{m}$ (Hodapp et al. 2012). EC 53 has a measured uncertainty greater than five times the fiducial standard deviation. No other sources have uncertainties greater than 2.5 times the fiducial standard deviation. Four of the brighter sources with $f_{m}>0.2$ Jy beam $^{-1}$, two each of protostars and disks, have marginal enhanced uncertainties, appearing slightly beyond the bulk of the ensemble in Figure 2. We investigate these outliers, along with EC 53, in Section 5 and present all five sources in Table 3.

An important concern when dealing with small-number statistics is the influence of outlier data points in our $\sim 12$ epochs. The occasional extreme data point will skew the measured uncertainty for the affected source unless the data are sigma-clipped, whereby data points well away from the mean are removed from the measurement set before statistical analysis. Additionally, given the large number of sources investigated, these extreme measurements happen relatively frequently within the sample even if rarely for a given object. To complicate this situation further, one of the primary goals of the JCMT Transient Survey is to uncover rare transient phenomena that will be mimicked by purely statistical large random deviations. Thus, much care is required to separate true stochastic behavior from statistical outliers due to random noise.

A detailed, robust investigation into the statistics of outlier points is beyond the scope of this initial results paper and will be more easily investigated as the number of epochs increases substantially, by a factor $\sim 2$, over the lifetime of the JCMT Transient Survey. Here we perform only a simple test for outlier points in the peak brightness data. For all epochs of each source, we calculate the deviation between the brightness measured and the mean brightness over all epochs in units of the measured standard deviation. We then remove (sigma-clip) those epochs that vary by more than two standard deviations and recompute both the mean and the standard deviation for the source. A total of 675 individual measurements $\left[f_{n}(i)\right]$ are removed by this process, representing about $5 \%$ of the entire measurement set as expected statistically for two-sigma 


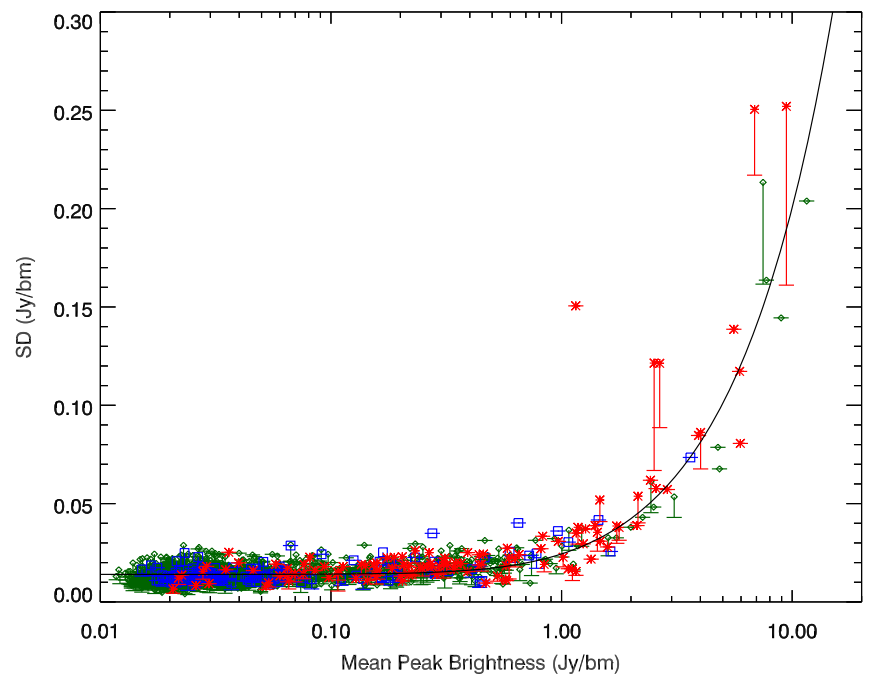

Figure 1. Scatter plot of measured mean peak brightness vs. measured standard deviation of peak brightness for all sources in the JCMT Transient Survey. Individual sources are colored green (diamonds) if they are starless, blue (squares) if they are associated with known disks, and red (stars) if they are associated with known protostars. The solid line denotes the fiducial model, $\mathrm{SD}_{\text {fid }}$, for the expected uncertainty in source brightness as a function of brightness (see Equation (1)). The symbols show the measured standard deviation in peak brightness when all epochs are used while the lower limits plot the result of sigma-clipping the measurement set (see the text).

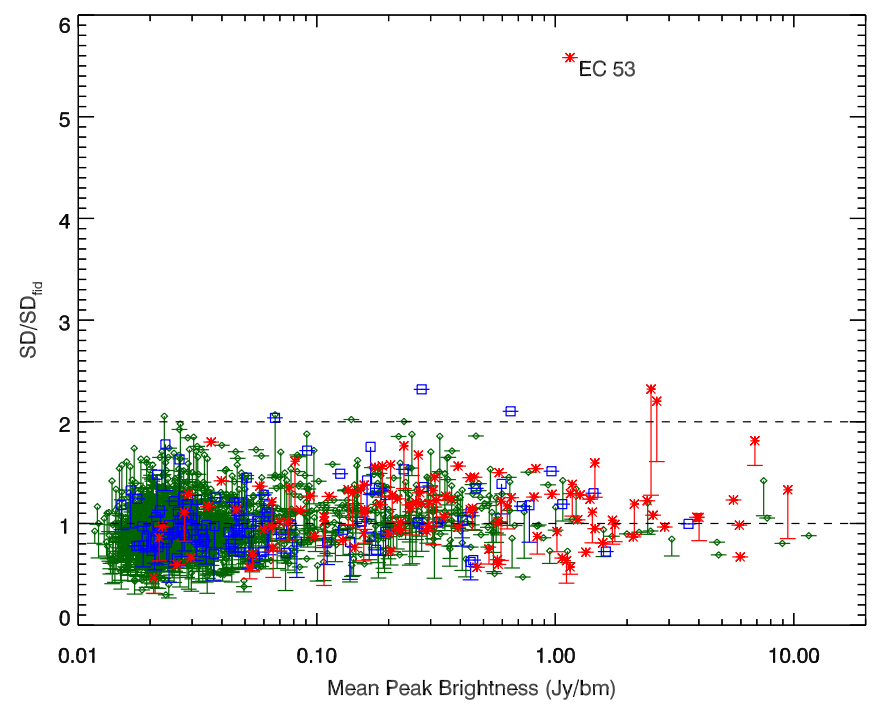

Figure 2. Scatter plot of measured mean peak brightness vs. measured standard deviation of peak brightness divided by the fiducial model for all sources in the JCMT Transient Survey. Individual sources are colored green (diamonds) if they are starless, blue (squares) if they are associated with known disks, and red (stars) if they are associated with known protostars. The dashed lines indicate unity and twice the fiducial expectation. The symbols show the results when all epochs are used while the lower limits plot the results after sigma-clipping the measurement set (see the text). Only one source stands out, EC 53 (Yoo et al. 2017), a known variable protostar in Serpens Main.

clipping of a normal distribution. In Figures 1 and 2, the downward error bars plot the change in the measured standard deviation for those sources where such outlier brightness measurements are removed. Note that the two protostars with initial measures of standard deviation somewhat larger than the ensemble fiducial regress significantly toward the standard deviation model when their outlier points, one per source, are removed (see Figure 2, Table 3, and Section 5). EC 53 and the two disk sources noted above are not influenced by the sigmaclipping process, i.e., no points are removed.

We have further analyzed the statistics of these 675 outlier brightness measurements and note that there is a significant skew in the distribution toward measurements higher than the mean, as opposed to outlier measurements for which the peak brightness was observed to decrease. While this is a possibly intriguing physical result, we caution that at least some of this may be due to data reduction artifacts, such as noise spikes in the original data set that have not been entirely removed before the map-making process. To be confident that the observed skew is due to a physical phenomenon associated with the observed submillimeter sources will require a more detailed investigation of where and when in each mapped region these outlier points are observed, including consideration of locations well away from known peaks. This investigation will be much easier to accomplish with the larger data set available at the end of the JCMT Transient Survey. We thus leave a full discussion of stochastic and transient phenomena to the survey summary paper and here only note that, of the 675 outlier data points uncovered, only seven are found to have offsets greater than than five times the source standard deviation. Based on normal statistics, the anticipated number of false positives with such large offsets is $\ll 1$ over the entire sample. Two of these extreme outliers are identified with sources brighter than $0.1 \mathrm{Jy}$ beam ${ }^{-1}$ - the two protostars discussed above and in Section 5 (see also Table 3).

In total, out of 1643 independent peaks analyzed, only EC 53 is found to have a measured standard deviation of brightness significantly larger than the expected fiducial. Thus, less than $0.1 \%$ of the ensemble peaks are found to be stochastically varying above the uncertainty in the survey measurements and less than $1 \%$ of the protostellar subsample show such variability. None of the $>1400$ peaks that are unassociated with known protostars shows significant evidence for stochastic variability.

\section{Secularity: Linear Variability Analysis}

The results of the previous section confirm that, at the level of calibration that we are able to achieve with the JCMT Transient Survey and the time range of observing, there is only one source at present that is undergoing very large variations in the peak brightness measurement at submillimeter wavelengths (EC 53; see also Mairs et al. 2017b; Yoo et al. 2017). The remaining sources all show scatter about the expected fiducial measure of standard deviation with only a hint that a few of these may be variable. Nevertheless, it is possible to investigate the range of (linear) secular brightness variations allowed by the measured brightnesses across all epochs and to search for statistically robust secular detections. Further, by comparing against the same brightness observations randomized in time order, we can also place limits on the degree of secular variation lurking within the ensemble. Given that we are interested in constraining the range of possible secular variability, this analysis includes those sources with a fiducial standard deviation of brightness (Equation (1)) of $<5 \%$, which corresponds to sources brighter than $0.35 \mathrm{Jy} \mathrm{beam}^{-1}$. Furthermore, since we are primarily interested in the statistical properties of the ensemble, we remove from the sample the strong detection, EC 53. Thus, as shown in Table 2, we are left with a sample of 150 peaks, of which 50 are associated with known protostars (Class 0/I) and 14 are associated with disks. 
Table 3

Potential Stochastic Variable Submillimeter Sources

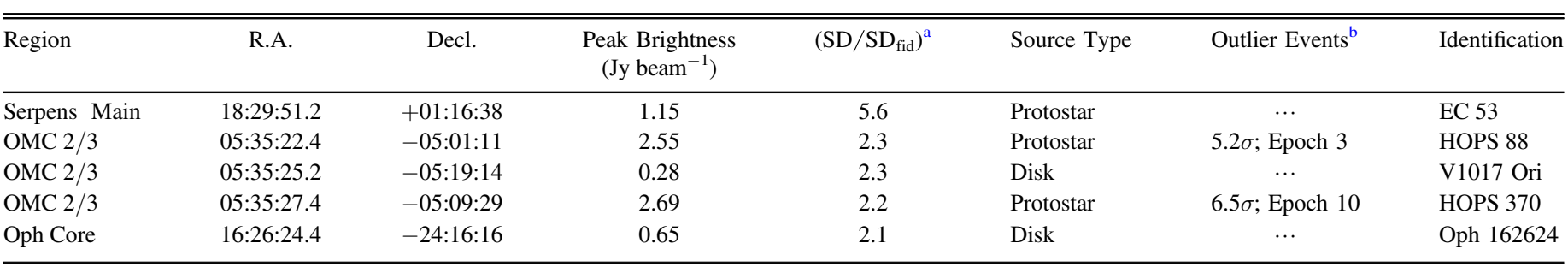

Notes.

${ }^{a}$ The fiducial model of the standard deviation of the flux density is described in Section 3.

${ }^{b}$ Shown are individual events greater than five times the measured standard deviation of the source.

Table 4

Secular Variability Analysis: Histogram Widths

\begin{tabular}{|c|c|c|c|c|c|c|c|c|}
\hline \multirow{2}{*}{ Ensemble } & \multicolumn{4}{|c|}{ Time-Ordered } & \multicolumn{4}{|c|}{ Randomized } \\
\hline & $\bar{S}^{\mathrm{a}}$ & $\sigma_{S}{ }^{\mathrm{b}}$ & $\overline{S / \Delta S}^{c}$ & $\overline{\sigma_{S / \Delta S} \mathrm{~d}}$ & $\bar{S}^{\mathrm{a}}$ & $\sigma_{S}^{\mathrm{b}}$ & $\overline{S / \Delta S} \bar{S}^{\mathrm{c}}$ & $\sigma_{S / \Delta S^{\mathrm{d}}}$ \\
\hline All bright sources & -0.006 & 0.023 & -0.27 & 1.25 & 0.000 & 0.022 & -0.02 & 1.11 \\
\hline Sample of protostars & -0.005 & 0.025 & -0.25 & 1.61 & 0.000 & 0.020 & -0.04 & 1.12 \\
\hline Clipped $^{\text {e }}$ sample of protostars & -0.006 & 0.020 & -0.27 & 1.11 & 0.000 & 0.020 & -0.04 & 1.04 \\
\hline
\end{tabular}

Notes.

${ }^{a}$ Measured mean value of the ensemble of slope measurements.

${ }^{b}$ Measured width of the ensemble of slope measurements.

${ }^{c}$ Measured mean value of the ensemble of slope divided by slope uncertainty measurements.

${ }^{\mathrm{d}}$ Measured width of the ensemble of slope divided by slope uncertainty measurements.

${ }^{\mathrm{e}}$ For the clipped sample we remove all sources with $|S / \Delta S|>3$ (see Section 4).

We begin by computing a least-squares linear fit to the source brightness across all measured epochs using the IDL routine linfit, which reproduces the fit and gammq routines described by Press et al. (1989). In this analysis we do not sigma-clip (see discussion in Section 3) and we further assume that all measurements for a given source have the same uncertainty. ${ }^{20}$ Thus, for each source, $i$, we derive a model fit, $f_{l}(i, t)$, that is linear over time, $t$, with two derived parameters: initial flux, $f_{0}(i)$ at time $t_{0}$, the time of the first epoch, and slope, $S(i)$, measured in fractional brightness change over a year:

$$
f_{l}(i, t)=f_{0}(i)\left(1+S(i)\left(t-t_{0}\right)\right) .
$$

Furthermore, in order to measure the relevance of any slopes departing from flat ( $S=0$; no change in brightness over time) we also compute the uncertainty in the slope, $\Delta S$, using the same IDL routine. ${ }^{21} \mathrm{We}$ then perform standard statistical analyses on the ensemble to determine the mean, standard deviation, and skewness of the set of measured slopes. These statistical measures of mean and standard deviation are presented in Table 4. In all cases the skewness is found to lie statistically close to zero (that is the distribution is well enough described by a Gaussian) and therefore is not included in the table.

\footnotetext{
${ }^{20}$ The observing strategy for the JCMT Transient Survey ensures that each epoch has roughly the same sensitivity (see Herczeg et al. 2017; Mairs et al. 2017a).

${ }^{21}$ The derivation of $\Delta S$ includes both the uncertainty in the measurement of $S$ and the uncertainty in the measurement of $f_{0}$ (Press et al. 1989).
}

The top panel of Figure 3 plots the histogram of the ensemble of measured slopes. The distribution is well fit by a Gaussian profile centered near zero with $\sigma_{S}=0.023$ (where $S= \pm 0.023$ corresponds to an increase or decrease in flux of $2.3 \%$ over a year; see Table 4 ), implying that a majority of the submillimeter peaks show little evidence for strong secular variability over timescales of a year. The protostar subset (red, hashed) follows the same overall distribution, with $\sigma_{S_{p}}=0.025$. While there are too few disks to obtain reliable statistical measures, the distribution shows no obvious disagreement with the full ensemble (not shown in the figure).

There are a few outlier slope measurements seen in the histogram (four with slope magnitudes larger than 0.06). To test whether these are statistically significant, we keep the same epoch dates and peak brightness measurements for each source but randomize the order in which the measurements are observed. In this way, the mean brightness and standard deviation for each source remain the same but any true secular variability will be removed. We are thus able to estimate the importance of false positive results for secular variability appearing in the wings of the distribution. The bottom panel of Figure 3 plots the randomized histogram of the ensemble of measured slopes after 100 independent randomizations for each source, and the parameters of the statistical fits are shown in Table 4. Again, a Gaussian profile fits the histogram very well, with an almost identical width to the ordered observations, $\sigma_{S}($ random $)=0.022\left(\sigma_{S_{p}}(\right.$ random $)=0.020$, slightly less than the width of the time-ordered protostellar sample). The fraction of randomized slope magnitudes larger than 0.06 (248 out of 15,000 , or $1.6 \%$ of the sample) is similar to the fraction of outlier sources in the top plot (four outliers, $2.7 \%$ of the 

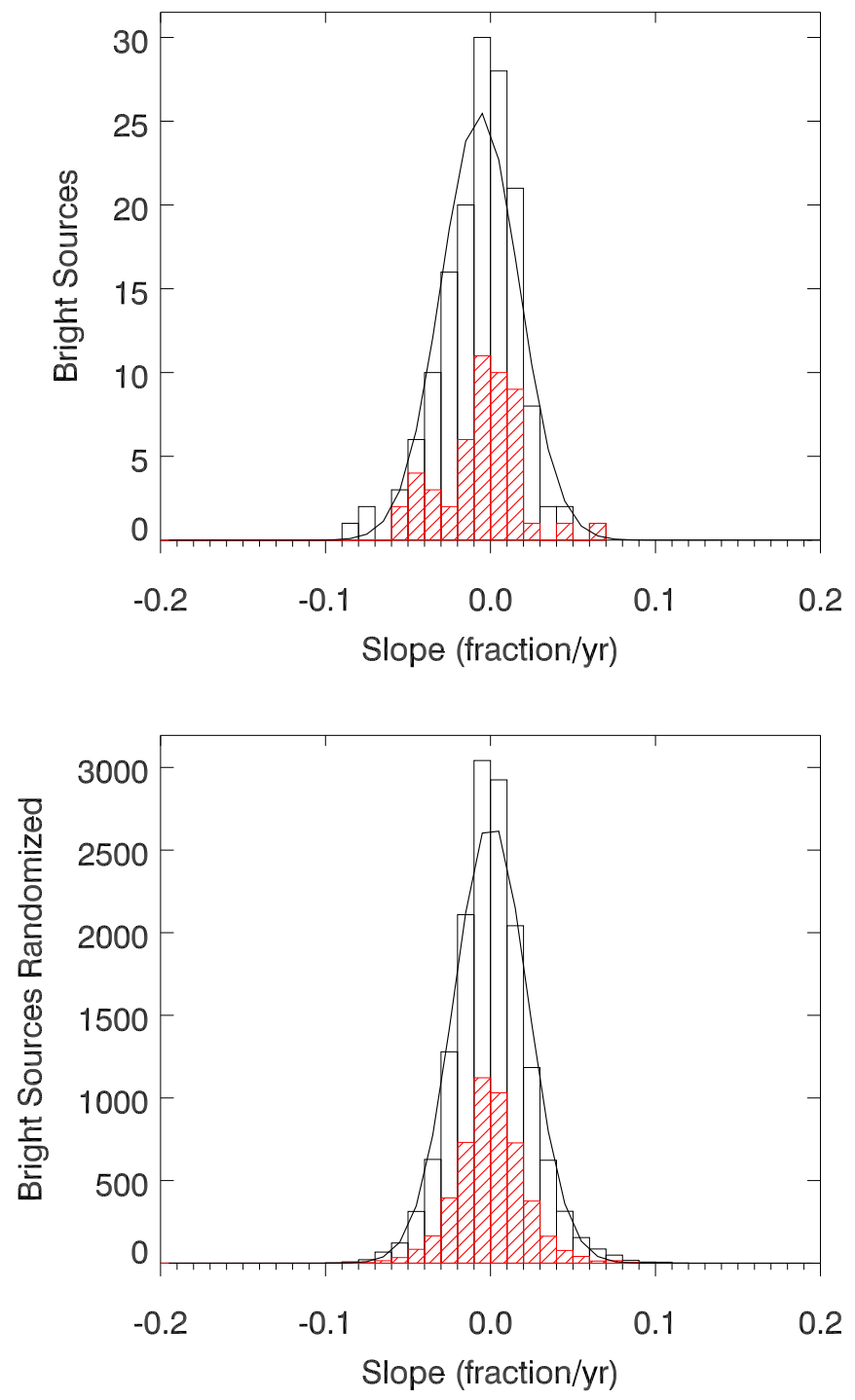

Figure 3. Histograms of the fractional change in peak brightness over a year for the ensemble of 150 bright sources in the JCMT Transient Survey. The 50 sources known to be associated with protostars are shown overplotted in red. Top panel: results from least-squares fitting to all epochs for each source. Bottom panel: results from least-squares fitting to all epochs for each source after randomly time-ordering the peak flux measurements 100 times. The parameters of the statistical fits are provided in Table 4. Note that the visible outlier measurements in the top panel are not significant (see the text).

sample), arguing that these outliers are not statistically significant.

Despite the lack of a clear signal in the measured slope histograms above, it is still possible that a subset of the bright sources may have statistically significant secular variation. We thus identify an additional goodness-of-fit metric for the slope measurements by taking the measured uncertainty in the slope and searching for those sources with $|S / \Delta S| \gg 1$. Again, we determine the statistical properties of the ensemble and use the randomized data set to test the likelihood of this metric revealing otherwise hidden secular variables (see Table 4). Figure 4 shows the distribution of $S / \Delta S$ for both the ordered measurements and 100 randomizations of each source. In both cases the histogram is well fit with $\sigma_{(S / \Delta S)} \sim 1$, as expected for a distribution dominated by uncertainty. More importantly, however, there exists a statistically relevant set of outliers in the ordered histogram, with $|S / \Delta S|>3$. We present these four sources, along with EC 53, in Table 5, where we also compute the false-positive expectation value derived from the randomized distribution. All five sources are protostellar (Class 0/I), yielding an observed rate of protostellar secular variability of $10 \%$. These sources are discussed individually in Section 5 and the significance of secular detections only within the protostellar sample is presented in Section 6. Recognizing that these outlier sources might skew the statistical analyses, we also provide results in Table 4 for the ensemble statistics after sigma-clipping the sources with $|S / \Delta S|>3$.

The outlier points in Figure 4 appear to be true deviants and not just an extension of a smooth distribution, although the limited statistics makes this challenging to quantify (see Section 4.1 below for additional comment). Furthermore, consideration of the light curves for these selected objects, presented in Section 5, suggests that the assumption of a purely linear secular variation is a significant oversimplification and that more complex analyses might uncover additional detail once the survey is complete. For the present, the limited number of epochs and the limited signal-to-noise ratio of the measurements versus the strength of any underlying secular variation make this linear investigation the appropriate first analysis.

Figure 5 shows the range in sensitivity to secular brightness changes for our sample as a function of source mean brightness. The typical uncertainty in the slope, $\Delta S$, is independent of source brightness for sources brighter than $1 \mathrm{Jy}^{\text {beam }}{ }^{-1}$ (see inset) and increases linearly for fainter sources. The distribution of uncertainties shows some scatter, since they depend on the individual source and the associated light curve. Robust detections of secular variability require $|S / \Delta S|>3$ and are shown in red in the main figure. There is no evidence in the figure that secular variables are biased toward either brighter or fainter submillimeter sources. However, given the individuality of the uncertainty measures, some sources are considered non-detections despite having steeper slopes than similar sources that are considered detections.

\subsection{Secular Variability: Toy Model}

Along with the individual outlier secular variables found in the preceding section, there may well be additional secular signal buried within the ensemble results presented in Figures 3, 4, and Table 4. To better understand the degree of secular variability allowed by the JCMT Transient Survey observations to date, and to predict the level of improvement expected over the lifetime of the survey, we design a simple toy source model and repeat the analysis from Section 4.

For simplicity, we assume an ensemble of 10,000 sources, each varying linearly in time with a slope, $S_{t}$, defining the fractional brightness change over a year, pulled from a normal distribution, $\sigma_{S_{t}}$. We further assume that these model sources are observed 12 times over 1.2 years with a regular cadence, approximately mimicking the available JCMT Transient Survey observations to date. Finally, we assume that the fractional uncertainty for each measurement for each source is $\Delta f / f=0.025$, the typical uncertainty of our observed sample. In the absence of this measurement uncertainty, the slope returned by the linear fit to each model source (Equation (2)) would match exactly the input value: $S=S_{t}$ (and $\sigma_{S}=\sigma_{S_{t}}$ ).

In Table 6 we present the statistical results of a linear leastsquares analysis for the toy model ensemble, both prior to and after time-randomization, for $\sigma_{S_{t}}=0.005,0.010$, and 0.020 . These results can be compared directly against the results from Section 4 and Table 4 . As can be seen in the table, for a large 

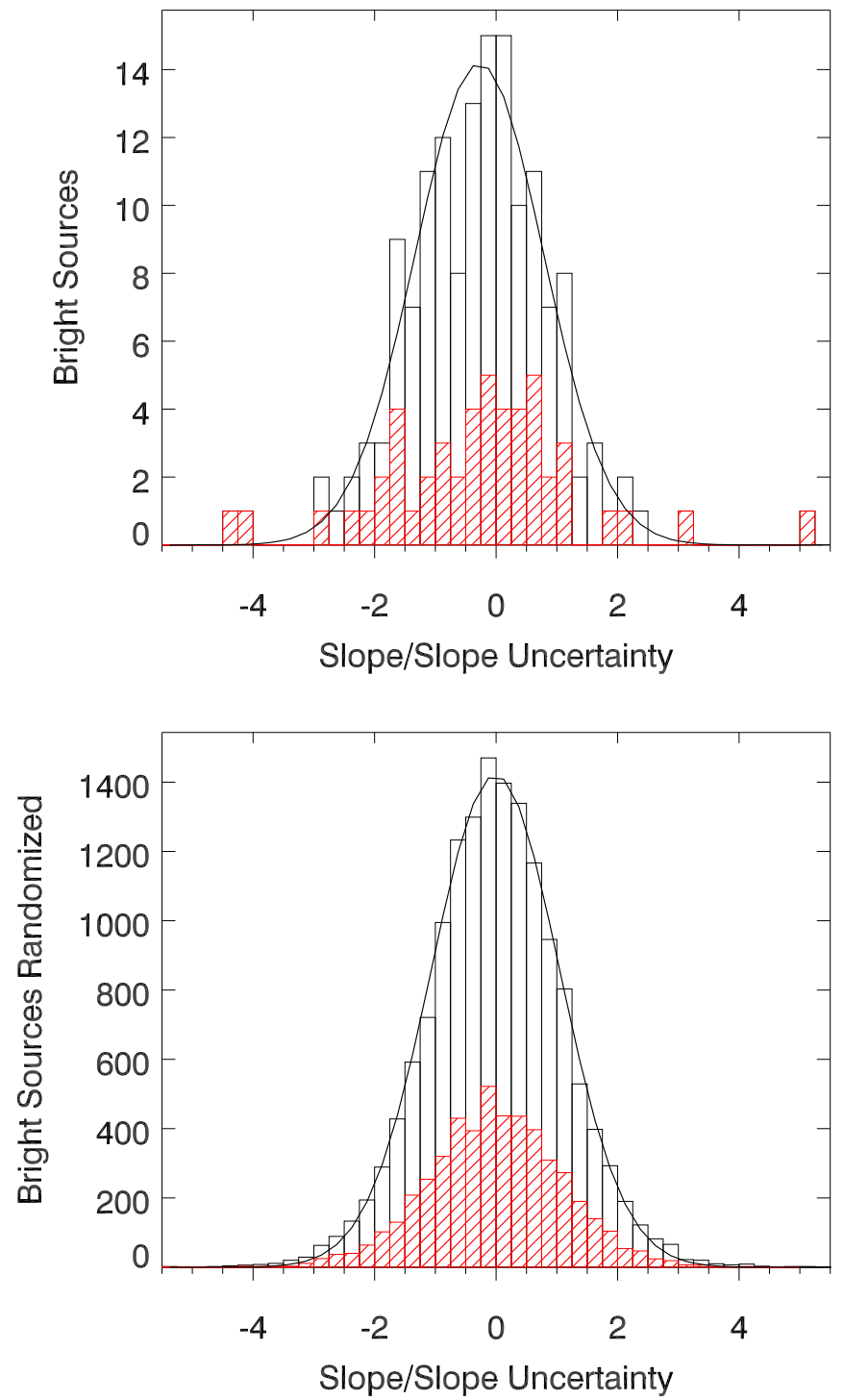

Figure 4. Histograms of the fractional change in peak brightness over a year divided by the uncertainty in the fractional change in peak brightness over a year for the ensemble of 150 bright sources in the JCMT Transient Survey. The 50 sources known to be associated with protostars are overplotted in red. Top panel: results from least-squares fitting to all epochs for each source. Note that the rightmost source in the top panel is SMM 10 in Serpens Main $(S / \Delta S=5.1)$. The source EC 53 is off the plot to the right $(S / \Delta S=7.9)$. Bottom panel: results from least-squares fitting to all epochs for each source after randomly time-ordering the peak flux measurements 100 times. The parameters of the Gaussian fits are provided in Table 4. Note that the outlier measurements in the top panel are significant (see Table 5 and the text).

value of $\sigma_{S_{t}}$ there is a significant difference in the width of the measured slope histogram between the original time-ordered data and the randomized data, with the distribution of the original time-ordered data being significantly broader than that of the randomized data. In all three model cases, the width of the measured slope for the randomized data remains similar to that for both the time-ordered and randomized data obtained by the JCMT Transient Survey to date, suggesting that the uncertainty assumptions of the toy model are reasonable. As the magnitude of $\sigma_{S_{t}}$ decreases, the measured slope distribution for the time-ordered data becomes narrower. The trends seen here are entirely as expected since the strength of the input slope within the toy model is proportional to $\sigma_{S_{t}}$ and becomes directly measurable in the time-ordered data as the secular signal becomes greater than the uncertainty in the individual measurements. For the randomized data, the input slope is significantly disrupted and thus there is little signal remaining to influence the histogram. The observed trend is similar for the histograms of measured slope in units of the measured slope uncertainty. For large values of $\sigma_{S_{t}}$ this width becomes significantly larger than unity for the time-ordered observations while the randomized distribution remains close to 1.1 (which is also the value obtained by the Transient Survey ensemble).

The toy model can be extended in order to predict the detection power of the JCMT Transient Survey after its full three years of observation. By increasing the number of observations to 30 and the time period to three years, we show in Table 6 that the difference in ensemble histogram widths between the time-ordered and random observations becomes much stronger for each value of $\sigma_{S_{t}}$. We thus conclude that at present we can rule out a Gaussian distribution of secular variations linear in time with $\sigma_{S_{t}}>0.02$ but that a distribution with $\sigma_{S_{t}}<0.01$ would be undetectable today. After the full three years of the survey, we expect to either measure $\sigma_{S_{t}}$ or rule out distributions with $\sigma_{S_{t}}>0.005$.

In each of these toy models, the normal distribution of linear slopes prevents there being a significant population of sources with large brightness variations, such as those observed by the JCMT Transient Survey (Table 5). This suggests that the five observed sources are indeed rare, statistical outliers. It should be recognized, however, that the toy models are meant to be representative rather than conclusive and that there remain many alternative distributions of secular variation, for example modified power laws, that might be able to fit the noisedominated ensemble measurements, similarly to the imposed normal distribution, while also allowing for a few rare outliers. Once the full survey is complete it will be appropriate to consider these more complex distributions.

\section{Individual Sources of Interest}

In the above investigation, we searched for stochastic and secular variables within the entire JCMT Transient Survey. In this section we discuss individually each candidate variable identified in this survey, along with several variables identified in Mairs et al. (2017b) and the submillimeter variable HOPS 383, identified by Safron et al. (2015). Where possible we compare those sources with robust secular fits with the measurements of brightness change obtained by Mairs et al. (2017b). Mairs et al. collated mean source brightnesses from the Transient Survey against archival JCMT Gould Belt Survey data (Ward-Thompson et al. 2007) to investigate variability over longer, 2-4 yr timescales, uncovering five strong candidates. As discussed in Section 6 and shown in Table 7, there is significant overlap between the sample of Mairs et al. (2017b) and the results from this paper. Mairs et al. (2017b) did not specifically consider stochastic variability, and the analysis technique used downweighted sources with large variations within one or both data surveys. Thus we are not able to compare directly the possible stochastic variables found here with that paper. At the end of this section we present light curves for three sources that are not found to vary in the present analysis - the brightest sources observed in the OMC 2/3 and Ophiuchus Core regions and the second brightest source in Serpens Main.

Figures 6-10 provide finding charts for each candidate variable source to show the location relative to other structures 
Table 5

Potential Secular Variable Submillimeter Sources

\begin{tabular}{|c|c|c|c|c|c|c|c|c|}
\hline Region & R.A. & Decl. & $\begin{array}{c}f_{0}{ }^{\mathrm{a}} \\
\left(\mathrm{Jy} \text { beam }^{-1}\right)\end{array}$ & $\begin{array}{c}S^{\mathrm{a}} \\
\left(\mathrm{yr}^{-1}\right)\end{array}$ & $S / \Delta S^{\mathrm{a}}$ & $\begin{array}{l}\text { Source } \\
\text { Type }\end{array}$ & $\begin{array}{l}\text { False-positive } \\
\text { Expectation }^{\mathrm{b}}\end{array}$ & Identification \\
\hline Serpens Main & $18: 29: 51.2$ & $+01: 16: 38$ & 0.95 & 0.28 & 7.9 & Protostar & $\ll 0.01$ & EC 53 \\
\hline Serpens Main & $18: 29: 52.0$ & $+01: 15: 50$ & 0.79 & 0.07 & 5.1 & Protostar & 0.01 & SMM 10 \\
\hline NGC 2068 & $05: 46: 31.0$ & $-00: 02: 32$ & 1.31 & -0.05 & 4.3 & Protostar & 0.08 & HOPS 373 \\
\hline Serpens South & $18: 29: 37.8$ & $-01: 51: 03$ & 0.68 & -0.05 & 4.1 & Protostar & 0.13 & IRAS $18270-0153$ \\
\hline Serpens Main & $18: 29: 49.8$ & $+01: 15: 20$ & 6.6 & 0.05 & 3.2 & Protostar & $1.33^{\mathrm{c}}$ & SMM 1 \\
\hline
\end{tabular}

Notes.

${ }^{\mathrm{a}}$ Fit parameters to the linear model described in Section 4.

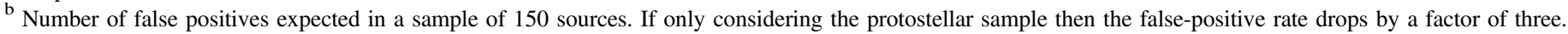

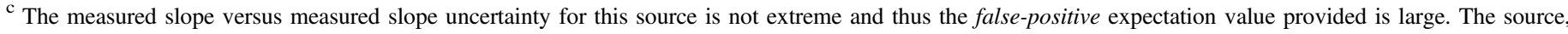

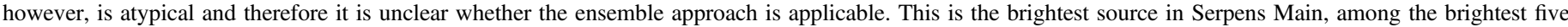
sources in the entire ensemble, and was found to vary in brightness between the GBS and Transient Surveys (Mairs et al. 2017b).

Table 6

Secular Variability Analysis: Histogram Widths in the Toy Model

\begin{tabular}{|c|c|c|c|c|c|c|c|c|}
\hline \multirow{3}{*}{ Toy Model } & \multicolumn{4}{|c|}{ Results for Present Survey } & \multicolumn{4}{|c|}{ Results after Full Survey } \\
\hline & \multicolumn{2}{|c|}{ Time-ordered } & \multicolumn{2}{|c|}{ Randomized } & \multicolumn{2}{|c|}{ Time-ordered } & \multicolumn{2}{|c|}{ Randomized } \\
\hline & $\sigma_{S}{ }^{\mathrm{a}}$ & $\sigma_{S / \Delta S^{\mathrm{b}}}$ & $\sigma_{S}{ }^{\mathrm{a}}$ & $\sigma_{S / \Delta S}{ }^{\mathrm{b}}$ & $\sigma_{S}{ }^{\mathrm{a}}$ & $\sigma_{S / \Delta S^{\mathrm{b}}}$ & $\sigma_{S}{ }^{\mathrm{a}}$ & $\sigma_{S / \Delta S^{\mathrm{b}}}$ \\
\hline$\sigma_{S_{t}}=0.010$ & 0.022 & 1.27 & 0.019 & 1.11 & 0.011 & 2.27 & 0.005 & 1.04 \\
\hline$\sigma_{S_{t}}=0.005$ & 0.020 & 1.15 & 0.019 & 1.11 & 0.007 & 1.47 & 0.005 & 1.03 \\
\hline
\end{tabular}

Notes.

${ }^{\text {a }}$ Standard deviation of $S$ measured for all members of the ensemble (see the text).

${ }^{\mathrm{b}}$ Standard deviation of $S / S_{e}$ measured for all members of the ensemble (see the text).

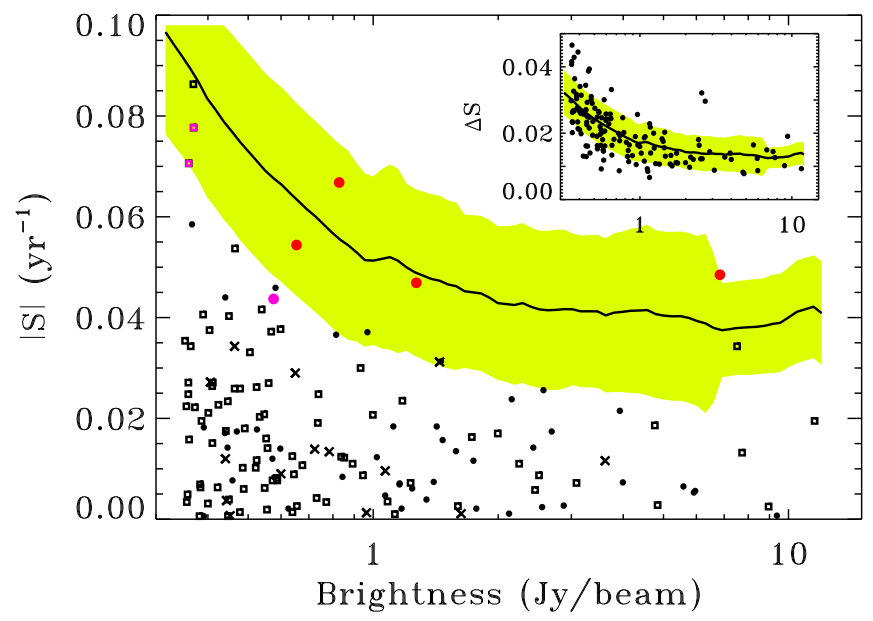

Figure 5. The distribution of the absolute value of the best-fit slope vs brightness (main plot), with an inset showing the uncertainty in slope, which increases to the faint end of our sample. At any given brightness, a wide range in uncertainties leads to large differences in the sensitivity of brightness changes. Filled circles denote known protostars, crosses denote disk sources, and squares represent starless cores. Symbols in red have $|S / \Delta S|>3$ and symbols in purple have $2.5<|S / \Delta S|<3$. The solid line shows a smoothed (0.4 dex in brightness) running average of the values that would yield a $3 \sigma$ detection for a typical source (the inset shows the $1 \sigma$ detection limit), with a standard deviation in the uncertainty displayed as the shaded yellow region. Some significant detections have slopes that are below the shaded region because the errors on those points are especially low, while other nondetections have slopes above the shaded region because of larger errors.

in the field. The following discussion also includes for each source a figure that shows the light curve and that describes the significance of secular and stochastic variability. A full population analysis is beyond the scope of this paper but is anticipated after the completion of our 3 yr survey. In general, most variables show some signs of an outflow, and several have been previously identified as candidates of ongoing FUorlike outbursts.

\subsection{Secular and Stochastic Variable: EC 53 (Serpens Main)}

EC 53 in Serpens Main (see Figure 6) was the first submillimeter variable revealed by the JCMT Transient Survey (see Yoo et al. 2017 for a more complete description) and is a known near-IR periodic variable (Hodapp et al. 2012). This source has the largest measure of stochastic variability in the Transient Survey (see Figure 2 and Table 3), as well as the strongest secular variability (see Table 5). Although the light curve should be periodic (see Yoo et al. 2017), the partial phasing over which the source has been observed for this investigation makes it stand out clearly as a linear variable (Figure 11). EC 53 was not recovered as a variable source by Mairs et al. (2017b), also due to the phasing of the GBS observations, although the GBS brightness is well described by the periodicity. That the periodicity of EC 53 is identified here as secular variability underscores the degenerate interpretations of other secular variables as either long-term trends or periodic variables.

\subsection{Secular Variable: SMM 10 (Serpens Main)}

SMM 10 in Serpens Main (Figure 6) has increased in brightness by $\sim 10 \%$ over the course of the survey (see Table 5 and Figure 12). Although the individual images of Serpens Main do not show any evidence of the variability of EC53 affecting other source measurements, monitoring this 

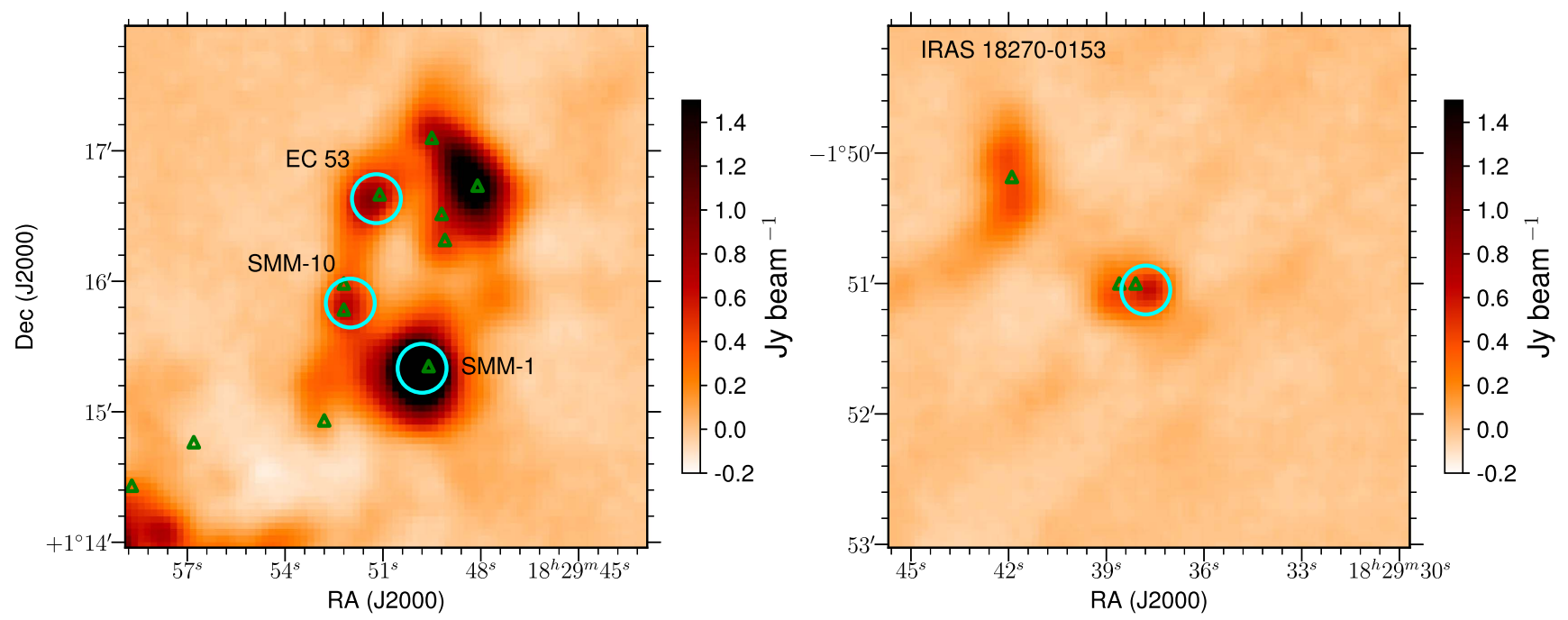

Figure 6. Potential secular variable sources in the Serpens Main (left panel) and Serpens South (right panel) molecular clouds overlaid on an $850 \mu \mathrm{m}$ SCUBA-2 map. The cyan circles indicate the location of the sources of interest and the green triangles indicate the positions of known protostars. Individual sources are discussed in Sections 5.1 (EC 53), 5.2 (SMM 10), 5.4 (IRAS 18270-0153), and 5.5 (SMM 1).

Table 7

Comparison of Identified Variable Sources

\begin{tabular}{|c|c|c|c|c|c|c|}
\hline \multirow[b]{2}{*}{ Region } & \multirow[b]{2}{*}{ Name } & \multicolumn{2}{|c|}{ Transient Analysis } & \multicolumn{2}{|c|}{$\begin{array}{c}\text { Transient-GBS } \\
\text { Analysis }\end{array}$} & \multirow[b]{2}{*}{ Comment } \\
\hline & & $(S / \Delta S)^{\mathrm{a}}$ & $\begin{array}{c}S \\
\left(\mathrm{yr}^{-1}\right)\end{array}$ & $\delta^{\mathrm{b}}$ & $\begin{array}{c}S \\
\left(\mathrm{yr}^{-1}\right)\end{array}$ & \\
\hline Serpens M & EC 53 & 7.9 & 0.28 & NA & NA & See Section 5.1 \\
\hline Serpens S & IRAS $18270-0153$ & 4.1 & -0.05 & 11.81 & -0.04 & Strong detection by both analyses \\
\hline NGC 2068 & HOPS 373 & 4.3 & -0.05 & 5.34 & -0.04 & Strong detection by both analyses \\
\hline Serpens M & SMM 1 & 3.2 & 0.05 & 6.85 & 0.02 & Strong detection by both analyses \\
\hline OMC $2 / 3$ & HOPS 383 & 3.0 & -0.04 & 4.17 & -0.03 & Moderate detection by both analyses \\
\hline NGC 1333 & Bolo 40 & 1.5 & -0.04 & 7.99 & -0.03 & Only source not identified with protostar \\
\hline NGC 1333 & IRAS 4A & $\ldots$ & $\ldots$ & 7.66 & 0.02 & Not detected by present analysis \\
\hline NGC 1333 & [LAL96] 213 & $\cdots$ & $\ldots$ & 8.31 & -0.09 & Not detected by present analysis \\
\hline Serpens M & SMM 10 & 5.1 & 0.07 & NA & NA & Source too faint for Transient-GBS detection \\
\hline
\end{tabular}

Notes.

${ }^{a}$ Sources with $|S / \Delta S|>4$ are robust against false positives within the entire ensemble (see Section 4). Those sources with $|S / \Delta S| \geqslant 3$ are strong candidates when treated as a special case.

${ }^{\mathrm{b}}$ Sources with $\delta>5$ are robust against false positives within the entire ensemble (see Mairs et al. 2017a). Those sources with $\delta>4$ are strong candidates when treated as a special case.

source over two full periods of EC 53, the 3 yr duration of the Transient Survey, will be helpful in disentangling any possible biasing by the presence of the nearby periodic variable. SMM 10 was too faint to be included in the variability analysis by Mairs et al. (2017b).

SMM 10 is considered a Class $0 / \mathrm{I}$ object based on the red spectral energy distribution and bright submillimeter continuum emission (e.g., Enoch et al. 2009b; Dunham et al. 2015). The source is associated with dense gas, which confirms that the protostar is embedded in an envelope (Lee et al. 2014). However, surveys of the mid-IR continuum and submillimeter CO 3-2 emission have found no evidence of outflows (e.g., Dionatos et al. 2010; Velusamy et al. 2014).

\subsection{Secular Variable: HOPS 373 (NGC 2068)}

HOPS 373 in NGC 2068 (see Figure 7) is a Class 0/I protostar, as measured from the red spectral energy distribution and bright submillimeter emission (e.g., Sadavoy et al. 2010; Furlan et al. 2016; Kirk et al. 2016). As noted in Section 2, this source is not included as a protostar in the original catalog of Megeath et al. (2012) but was noted by Stutz et al. (2013) as a PACS Bright Red Source (PBRS). The robust detection found here (Table 5) shows a step-like decline in brightness a few months after the start of our Transient Survey. HOPS 373 was also found to decline in brightness between the GBS and Transient Surveys but the significance of the decline, $\delta \sim 4$ (see Section 6 and Table 7), was just under the threshold for inclusion in the robust sample (see Mairs et al. 2017b for details). The submillimeter light curve and histograms showing the likelihood of this source having secular variability are shown in Figure 12.

HOPS 373 has a bright, wide-angle outflow with bright emission in highly excited far-IR molecular lines (Manoj et al. 2016; Tobin et al. 2016b), and may also be associated with a 


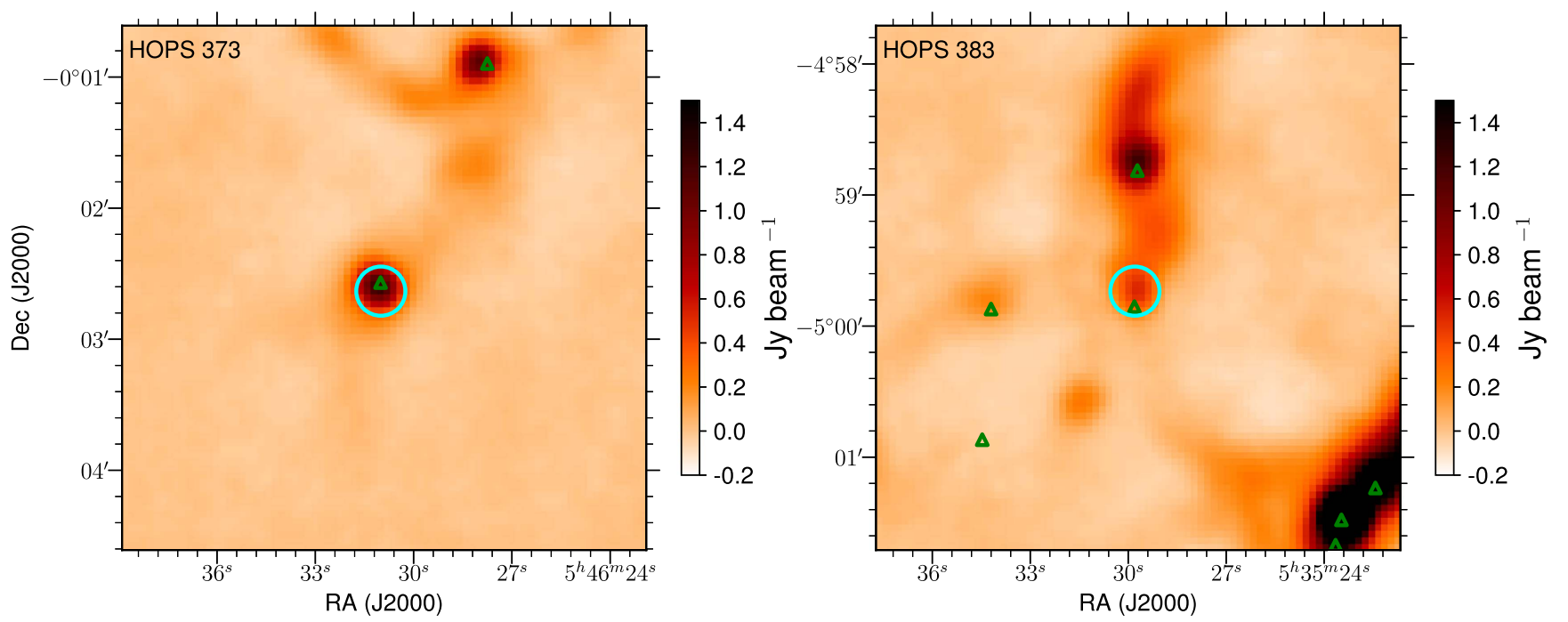

Figure 7. Same as Figure 6 but for identified variable sources in the Orion B (left panel) and Orion A (right panel) molecular clouds. Individual sources are discussed in Sections 5.3 (HOPS 373) and 5.10 (HOPS 383).
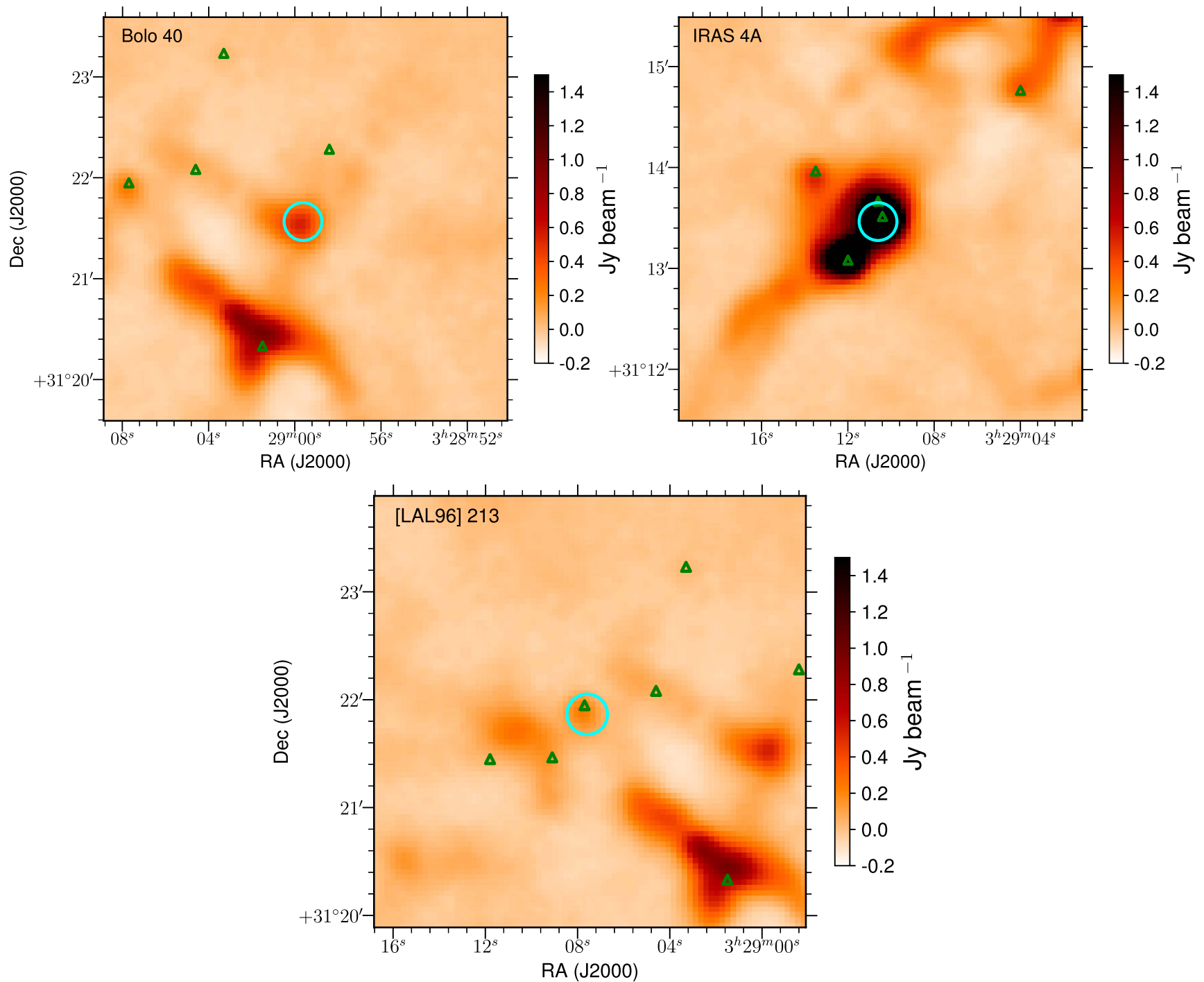

Figure 8. Same as Figure 6 but for identified variable sources in the Perseus molecular cloud. Individual sources are discussed in Sections 5.11 (Bolo 40 ), 5.12 (IRAS 4A), and 5.13 ([LAL96] 23). 

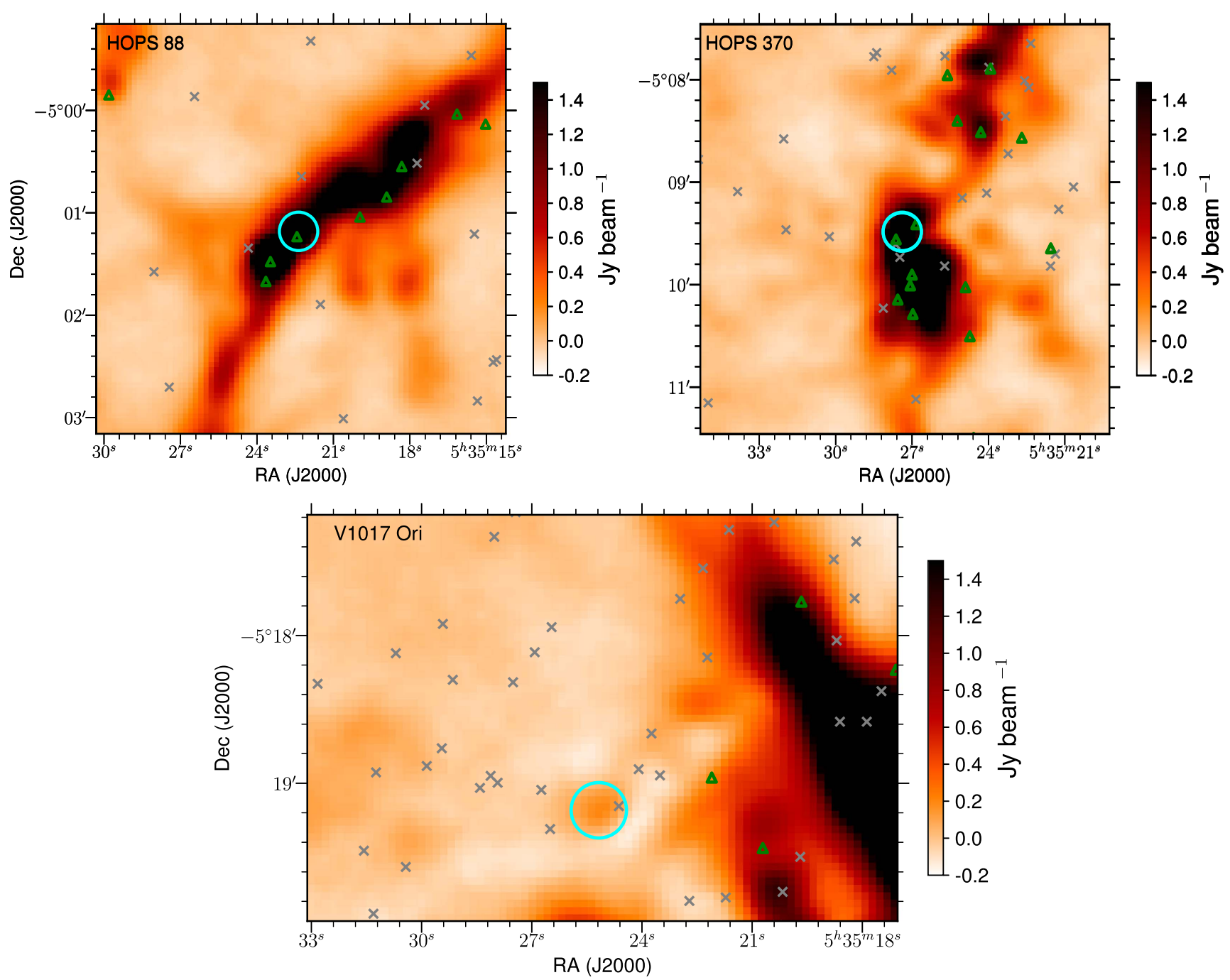

Figure 9. Potential stochastic variable sources in the Orion A molecular cloud overlaid on an $850 \mu \mathrm{m}$ SCUBA-2 map. The cyan circles indicate the location of the sources of interest, the green triangles indicate the positions of known protostars, and the gray crosses indicate the positions of known disks. The lower bound of the V1017 Ori panel (bottom) is the edge of the Transient Survey OMC 2/3 region. Individuals sources are discussed in Sections 5.6 (HOPS 370), 5.7 (HOPS 88 ), and 5.8 (V1017 Ori).

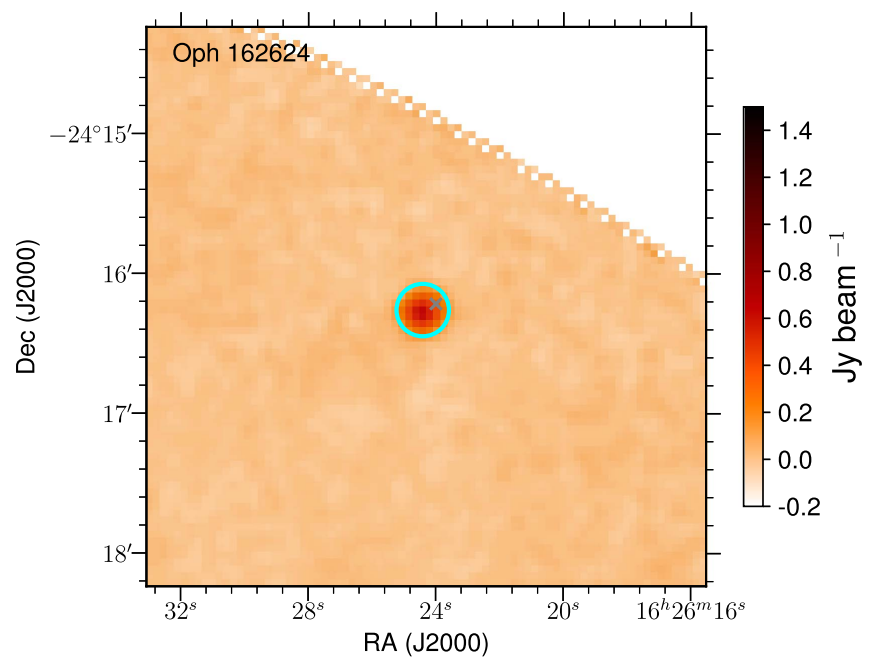

Figure 10. Same as Figure 9 but for the stochastic variable source in the Ophiuchus molecular cloud. Oph 162624 is discussed in Section 5.9 . nearby Herbig-Haro object with an $\mathrm{H}_{2} \mathrm{O}$ maser (Haschick et al. 1983; Yu et al. 2000).

\subsection{Secular Variable: IRAS 18270-0153 (Serpens South)}

The source at 18:29:37.8-01:51:03 in Serpens South is located $\sim 5^{\prime \prime}$ from IRAS 18270-0153, within our uncertainty beam (see Figure 6). This source has a robust decrease in brightness between the GBS and Transient Surveys (Mairs et al. 2017b). Here, we also find the source fading with time (Figure 12 and Table 5), and note that this fade may be either gradual or step-like.

IRAS 18270-01530 is an embedded object with bright millimeter-continuum emission (e.g., Maury et al. 2011) and an outflow seen in near-IR $\mathrm{H}_{2}$ and low- $J \mathrm{CO}$ emission, but not in excited far-IR molecular emission (Zhang et al. 2015; Mottram et al. 2017). IRAS 18270-01530 is considered an FUor candidate based on deep $\mathrm{CO}$ absorption in the $K$ band (Connelley \& Greene 2010). Thus, the submillimeter fading may be consistent with a long-term decay of the outburst. 

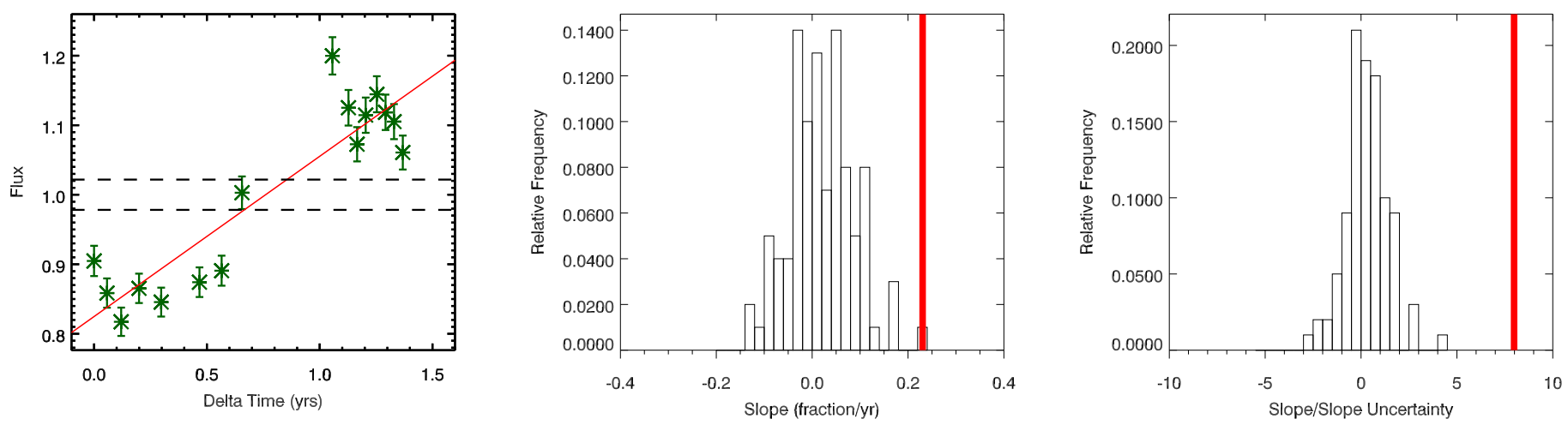

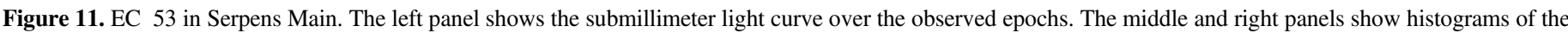

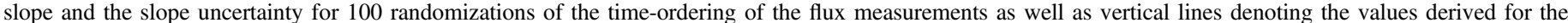
observed light curve.

\subsection{Secular Variable: SMM 1 (Serpens Main)}

Serpens SMM 1 in Serpens Main (see Figure 6) is the brightest submillimeter source in Serpens. Although SMM 1 has the highest likelihood of being a false positive among the five protostellar variables identified here (Table 5), the comparison between the GBS data and our Transient Survey yields a similar increase in brightness with time (Mairs et al. 2017b). The submillimeter light curve shown in Figure 12 suggests additional structure beyond a simple linear increase.

SMM 1 is the prototypical intermediate-mass Class 0 star with a massive, $8 M_{\odot}$ envelope (e.g., Enoch et al. 2009a) and bolometric luminosity of $\sim 69 L_{\odot}$ (Dunham et al. 2015). The outflow from the source includes a jet, surrounded by an ionized outflow and a molecular outflow (e.g., Goicoechea et al. 2012; Hull et al. 2016).

\subsection{Stochastic Variable: HOPS 370 (OMC 2/3)}

HOPS 370 (OMC 2 FIR 3) in OMC 2/3 (see Figure 9) is one of three potential stochastic variable protostars found in this investigation (Table 3). The object is considered an intermediate-mass Class I object, with a bolometric luminosity of $361 L_{\odot}$ (e.g., Fischer et al. 2017). Its submillimeter light curve (Figure 13) shows that the standard deviation of the mean brightness of the source is dominated by a single epoch, during which the source appears to increase in brightness by $\sim 10 \%$. The protostar is likely associated with the optically bright companion, V2467 Ori, located $\sim 3^{\prime \prime}$ away.

Light curves of V2467 Ori at 3.6 and $4.5 \mu \mathrm{m}$ from Spitzer/ IRAC show that the combined emission from these two sources is generally stable (Morales-Calderón et al. 2011). The source also has stable far-IR emission to within $10 \%$ in six epochs obtained over 1.5 months with Herschel/ACS (Billot et al. 2012). The source is associated with bright outflow emission (e.g., Yu et al. 2000; Takahashi et al. 2008; Kang et al. 2013).

\subsection{Stochastic Variable: HOPS 88 (OMC 2/3)}

HOPS 88 (OMC 3 MMS 5) in OMC 2/3 (see Figure 9) is another of three potential stochastic variable protostars found in this investigation (Table 3). Like HOPS 370, the submillimeter light curve of HOPS 88 (Figure 13) shows that the standard deviation of the mean brightness of the source is dominated by a single epoch, albeit a different one than for HOPS 370, during which the source appears to increase in brightness by $\sim 10 \%$. HOPS 88 shows some variability in 3.6 and $4.5 \mu \mathrm{m}$ continuum emission (Morales-Calderón et al. 2011), and varied by $19 \%$ in six epochs of far-IR continuum emission (Billot et al. 2012). HOPS 88 is associated with dense gas and CO outflows (e.g., Takahashi et al. 2008, 2009).

\subsection{Stochastic Variable: V1017 Ori (OMC 2/3)}

V1017 Ori in OMC 2/3 (see Figure 9) is one of two potential stochastic variable disk sources found in this investigation (Table 3). The centroid of the submillimeter emission source is $10^{\prime \prime}$ from the optical/near-IR source, within a single submillimeter beam but too distant to be confident in the association. The submillimeter light curve of V1017 Ori (Figure 13) reveals that no individual measurement is responsible for the enhanced uncertainty in its measured brightness.

The spectral type M3.5 for V1017 Ori implies a mass of $\sim 0.2-0.3 M_{\odot}$ (Hillenbrand 1997; Da Rio et al. 2016). Optical spectra and photometry of V1017 also show strong $\mathrm{H} \alpha$ emission, indicating ongoing accretion (e.g., Manara et al. 2012). Variability in the near-IR and in Spitzer/IRAC 3.6 and $4.5 \mu \mathrm{m}$ light curves is likely caused by changes in the inner disk structure (Carpenter et al. 2001; Morales-Calderón et al. 2011).

\subsection{Stochastic Variable: Oph 162624 (Ophiuchus)}

Oph 162624 (YLW 32; Elia 2-24) in Ophiuchus (see Figure 10) is one of two potential stochastic variable disk sources found in this investigation (Table 3 ). The submillimeter light curve (Figure 13) reveals that no individual measurement is responsible for the enhanced uncertainty in its measured brightness.

The brightness of the disk has made it a prominent object for high-resolution studies (e.g., Andrews \& Williams 2007). The star has an effective temperature and luminosity that imply a central mass of $\sim 1.5 M_{\odot}$, and the emission lines from the star indicate continued strong accretion (Manara et al. 2015; Rigliaco et al. 2016).

\subsection{Literature Variable: HOPS 383 (OMC 2/3)}

HOPS 383 in OMC 2/3 (at 5:35:29.67-4:59:37.25; see Figure 7) was found to decrease in brightness between the GBS Survey and the Transient Survey (Mairs et al. 2017b), albeit 

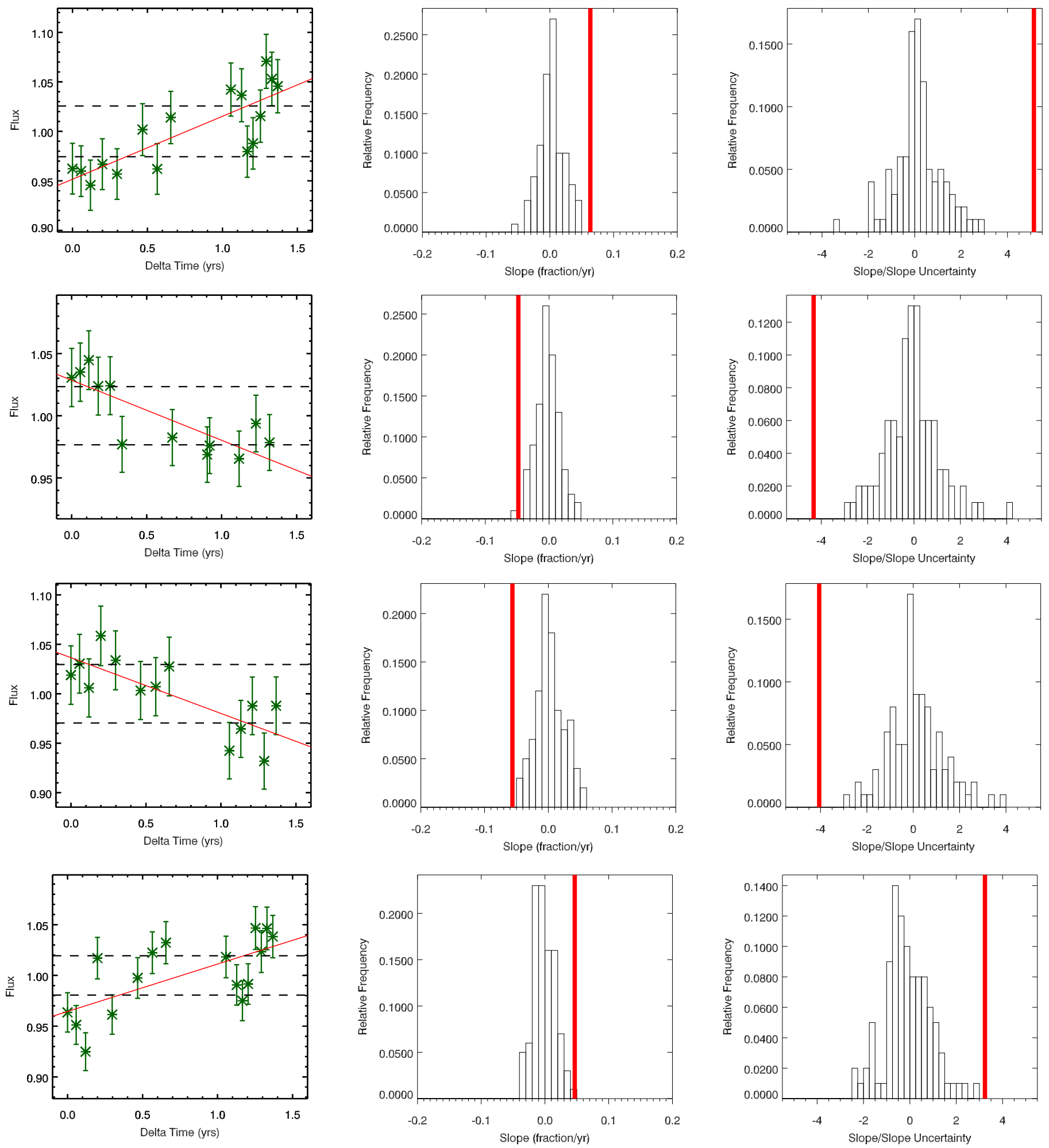

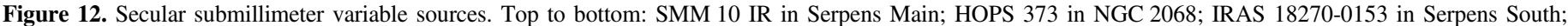

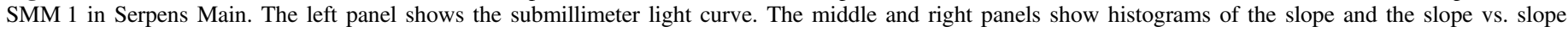

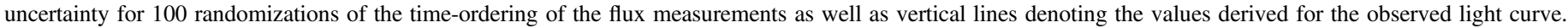

with a marginal significance. The submillimeter light curve (Figure 14) across only the Transient Survey also shows moderate evidence of a decrease, at a similar rate as found by Mairs et al. (2017b). Previously, this object produced a remarkable outburst in IR and submillimeter wavelengths (Safron et al. 2015). The fade in submillimeter continuum emission is in the same direction as the dramatic fade seen in the near-IR (Fischer \& Hillenbrand 2017), but is much more modest, indicating that either the near-IR suffers from increasing extinction or the time delay between any changes in the near-IR and submillimeter emission is much longer than expected. 

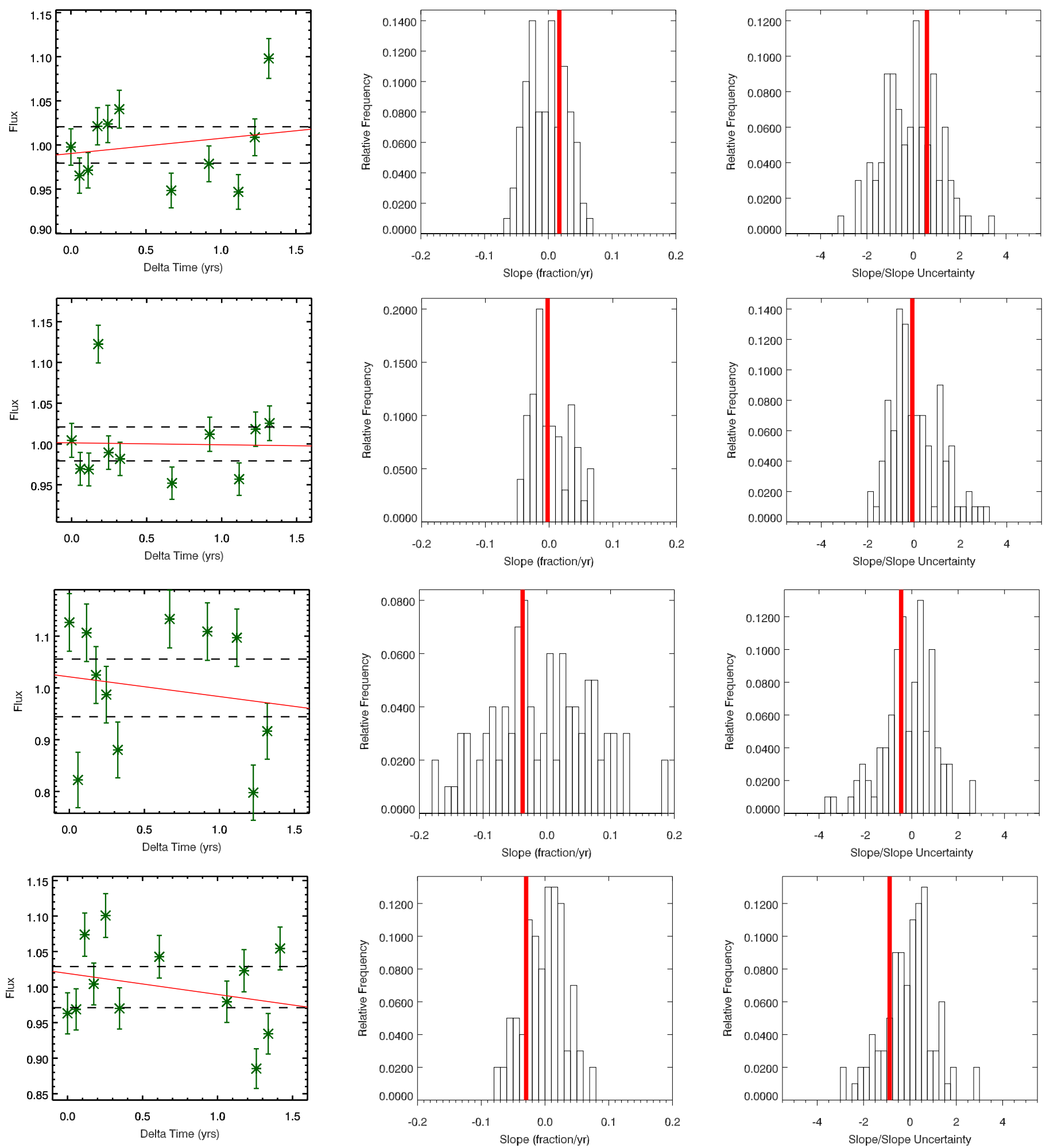

Figure 13. Stochastic submillimeter variable sources. Top to bottom: HOPS 370 in OMC 2/3; HOPS 88 in OMC 2/3; V1017 Ori in OMC 2/3; Oph 162624 in Ophiuchus. The left panel shows the submillimeter light curve. The middle and right panels show histograms of the slope and the slope vs. slope uncertainty for 100 randomizations of the time-ordering of the flux measurements as well as vertical lines denoting the values derived for the observed light curve.

\subsection{Literature Variable: Bolo 40 (Perseus)}

Bolo 40 in Perseus (3:28:59.86+31:21:33.09; see Figure 8) was found to decrease gradually, by about $3 \%$ per year, between the GBS Survey and the Transient Survey (Mairs et al. 2017b). The submillimeter light curve (Figure 14) across only the Transient Survey shows only a hint of decline, which would be expected if the source continued to dim in brightness slowly. Bolo 40 is the only potentially variable source discussed in this paper that is not known to be associated with either a protostar or a disk. 

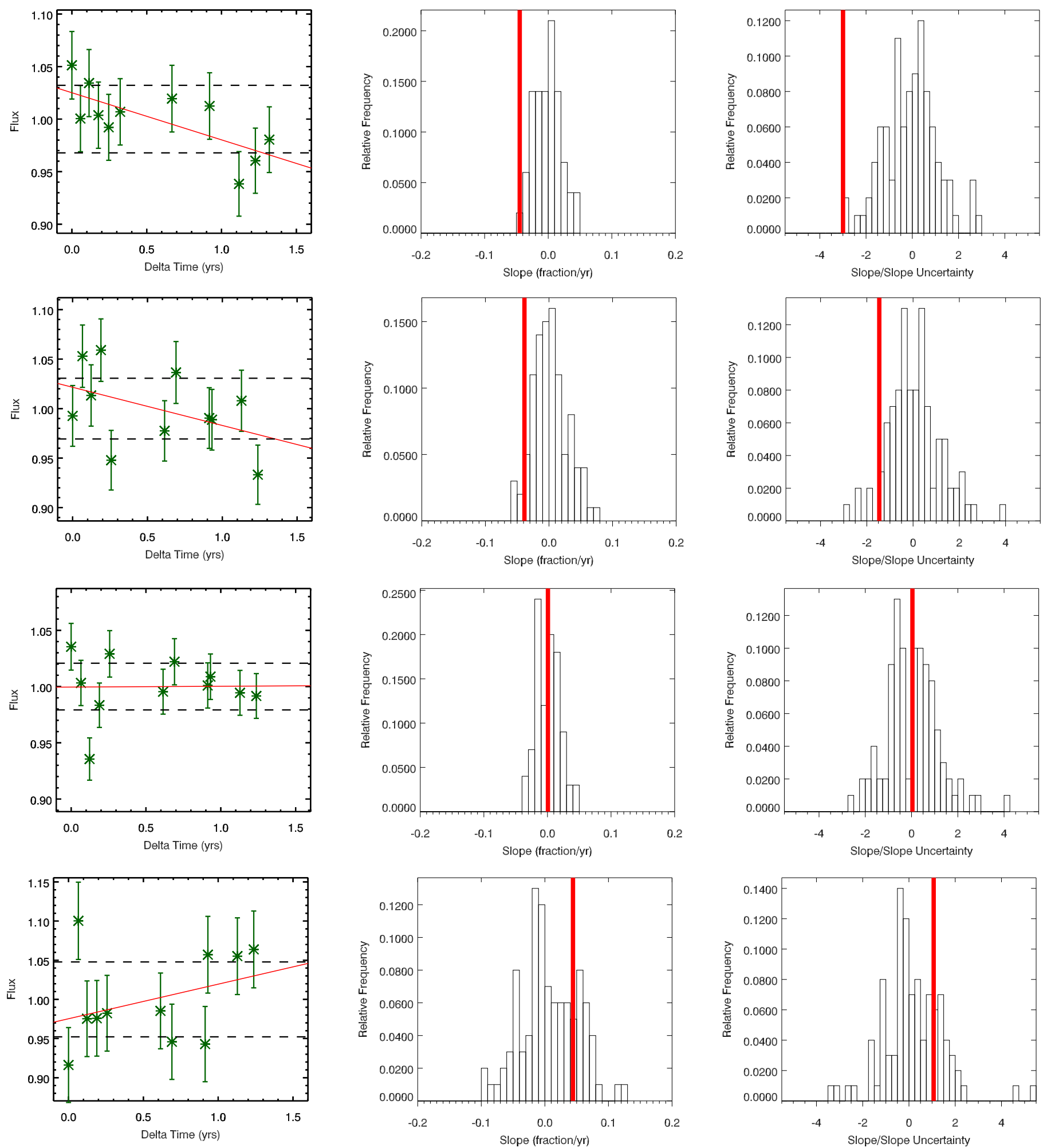

Figure 14. Literature variable sources. Top to bottom: HOPS 383 in OMC 2/3; Bolo 40 in Perseus; NGC 1333 IRAS 4A in Perseus; LAL96 213 in Perseus. The left panel shows the submillimeter light curve. The middle and right panels show histograms of the slope and the slope vs. slope uncertainty for 100 randomizations of the time-ordering of the flux measurements as well as vertical lines denoting the values derived for the observed light curve.

\subsection{Literature Variable: NGC 1333 IRAS 4A (Perseus)}

NGC 1333 IRAS 4A in Perseus (at 3:29:10.42+31:13:30.63; see Figure 8 ) was found to increase slowly, by about $2 \%$ per year, between the GBS Survey and the Transient Survey (Mairs et al. 2017b). The submillimeter light curve (Figure 14) across only the Transient Survey, however, shows no clear evidence of a secular variation, as expected if the rise in brightness remained slow.

\subsection{Literature Variable: LAL96 213 (Perseus)}

LAL96 213 in Perseus (at 3:29:07.66+31:21:54.05; see Figure 8) was found to decrease substantially between the GBS 

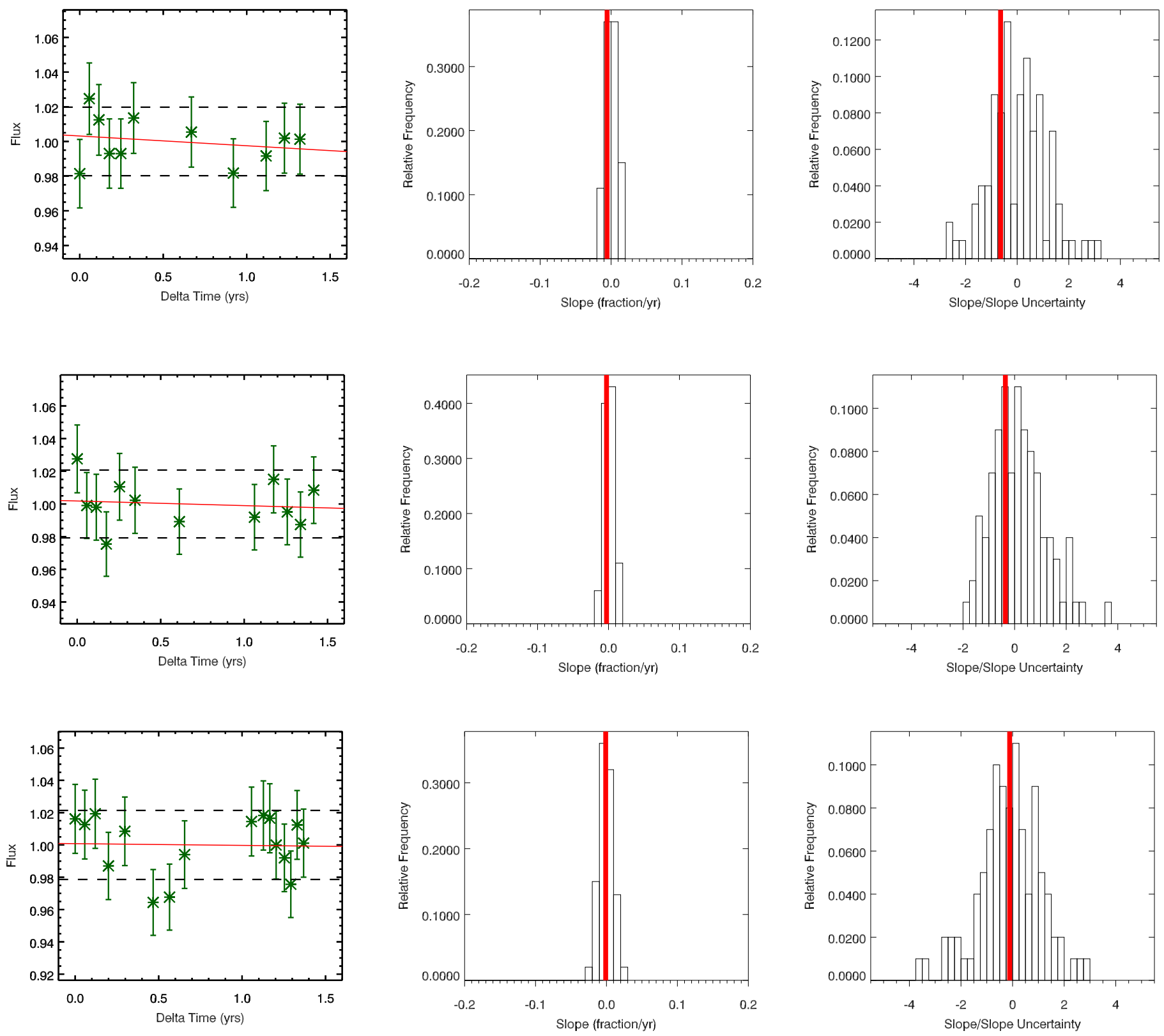

Figure 15. Non-variable sources. Top to bottom: HOPS 64 in OMC 2/3 (also known as OMC 2 FIR 4); SMM J162628-24235 in the Ophiuchus Core; SH $2-68$ N in Serpens Main. The left panel shows the submillimeter light curve. The middle and right panels show histograms of the slope and the slope vs. slope uncertainty for 100 randomizations of the time-ordering of the flux measurements as well as vertical lines denoting the values derived for the observed light curve.

Survey and the Transient Survey (Mairs et al. 2017b). The submillimeter light curve (Figure 14) across only the Transient Survey, however, does not show any evidence of a secular decrease, suggesting that the rate of decline has tapered off over the last 18 months. As shown in the right panels of Figure 14, the slope fit to the left panel is not significant.

\subsection{A Sample of Non-variable Sources}

Figure 15 provides light curves for three sources that have not been observed to vary during the Transient Survey so far. These are not random objects, but rather the brightest sources in OMC 2/3 (HOPS 64 also known as OMC 2 FIR 4; Furlan et al. 2014) and the Ophiuchus Core (SMM J162628-24235; Jørgensen et al. 2008), as well as the second brightest source in Serpens Main (SH 2-68 N; Winston et al. 2007). Note that the brightest source in Serpens Main, SMM 1, was found to vary secularly (Section 5.5). These sources are presented as examples of how flat the light curves can be when the sources are bright and the measured uncertainties are small (see, for example, the middle panel in each figure, which plots the range of slopes allowed by randomizing the time-ordering of the measurements).

\section{Discussion}

Our search for secular changes in submillimeter brightness over the lifetime of the JCMT Transient Survey has uncovered five robust variables (see Table 5). Another source, HOPS 383, is likely to be a secular submillimeter variable (Section 5.10). These numbers can be compared against the total number of protostars observed with submillimeter peaks $>0.35 \mathrm{Jy}$ beam ${ }^{-1}$ (51; Table 2). We therefore find that few protostars vary with large, $>10 \%$, amplitudes in the submillimeter continuum over 
timescales of a year, but $\sim 10 \%$ of them vary by $\sim 5 \%$ over a year. This later fraction is in agreement with the result obtained by Mairs et al. (2017b), who compared the mean Transient Survey brightnesses of sources against the mean brightness measured from data obtained two to four years earlier by the JCMT Gould Belt Survey (Ward-Thompson et al. 2007). As such, it would appear that moderate variability of protostars at submillimeter wavelengths is relatively common. These early results will be considerably strengthened over the lifetime of the Transient Survey, as discussed in Section 4.1. Significant numbers of sources with linear brightness changes as small as $2 \%$ per year should be directly observable at that time.

All five secular variables identified in this paper are protostellar, despite our sample including twice as many bright starless cores as protostars (see Section 4). While the brightness distribution of starless cores is skewed toward lower values than the protostellar sample, the bright end contains similar numbers of starless cores and protostars (see Figure 5). Thus, to first order the two samples are quite similar over the range of brightnesses for which secular variability was uncovered in the protostellar sample. As such, assuming that both the protostar and prestellar samples have the same underlying variability properties, the likelihood of all five sources being drawn from the protostellar sample is about $3 \%$. While not impossible, it is much more likely that the prestellar sample is not varying at the same level as the protostellar sample. This difference is expected because prestellar cores do not have any known source of energy to provide a time-dependent brightness at submillimeter wavelengths on timescales of a month to a year.

The lack of strong stochastic variables within the present sample, excluding EC 53, suggests that significant brightness changes $>10 \%$ rarely occur over very short (monthly to yearly) timescales for the typical protostar or disk source. Indeed, the only source observed to have a large standard deviation in its brightness measurements, EC 53, is best fit as an 18 month periodic variable with a large amplitude, rather than as a randomly varying source. Perhaps more interesting are the possible additional periodic variables within the present data set with low amplitudes below our present detection limit, which will require time-domain Fourier analyses to uncover. For such sources, a greater number of epochs and a larger time range to compare against will be extremely beneficial.

Many of the secular variables identified in this paper show light curves that appear to be more complicated than a simple linear variation in time, see Section 5. Nevertheless, three of the five sources identified here as secular variables are also seen to vary strongly by Mairs et al. (2017b) as shown in Table 7 . One of the two non-detections, EC 53, was excluded from the variable source list of Mairs et al. precisely because of the large standard deviation of its individual brightness measurements. The other source, SMM 10, was too faint for the analysis of Mairs et al. Similarly, of the five robust variable detections found by Mairs et al. ( $\delta>6$ in Table 7$)$, we recover two as robust secular variables and one more as possibly varying. The larger time separation, two to four years, for the analysis of Mairs et al. allowed for the recovery of sources varying with lower amplitudes than detectable over the first 18 months of the Transient Survey, and this likely accounts for the poorer match between sources identified by Mairs et al. (2017b) and this paper. An additional ninth source, the known submillimeter variable HOPS 383 , is found to be a likely dimming variable by both analyses. For all of these sources, the sign of the brightness slope (rising or dimming) is always the same between the two analyses, when measurable (see Table 7). The agreement between these two independent investigations suggests that the assumption of a long-term, multi-year, timescale underlying the light curve is reasonable. As with the stochastic discussion above, future observations of these sources will help to identify any quasi-periodic underlying nature.

It is important to recognize that the submillimeter continuum emission is likely responding to the change in the dust temperature within the protostellar envelope and accretion disk, and is not necessarily directly proportional to the underlying change in accretion luminosity of the central source (Johnstone et al. 2013; Yoo et al. 2017). Detailed modeling is required to determine the exact relationship between the change in submillimeter brightness and the change in the accretion luminosity, taking into account the range of dust temperatures in the disk and envelope as well as the importance of external heating of the outer envelope. Mairs et al. (2017b) consider these issues and conclude that the amplitude of the change in the accretion rate should scale somewhere between the amplitude of the submillimeter brightness change and the fourth power of the submillimeter brightness change. Thus, in rough agreement with the numbers computed by Mairs et al., we find that about $10 \%$ of the protostars in the Transient Survey are undergoing variations in mass accretion between $5 \%$ and $20 \%$ over the course of a year. Such values lie intermediate between the numerical results for accretion instability driven by magnetorotation (Bae et al. 2014) and those driven by large-scale modes within a gravitationally unstable disk (Vorobyov \& Basu 2010), both of which are presented by Herczeg et al. (2017). However, neither of these models was intended to be used for measurements on such short timescales. Nevertheless, this discussion shows that results from the Transient Survey will help to constrain the next generation of numerical models of accretion disks.

This interpretation assumes that the variability in submillimeter emission is caused by a change in the protostellar luminosity. However, the two stochastic variables with single discrepant points may instead have individual bright epochs caused by radio synchrotron flares (e.g., Bower et al. 2003; Forbrich et al. 2017). In these situations the spectral index in the optically thick regime would be flat or inverted such that the flux at centimeter wavelengths would be comparable to or lower than that in the submillimeter region. As an example, the young stellar object GMR A in Orion was observed to brighten to $100 \mathrm{mJy}$ at $3 \mathrm{~mm}$ wavelengths (Bower et al. 2003). Since these magnetic flares typically last for hours, they would be unable to explain the other submillimeter variables identified in this paper.

A curious result from the present survey is the number of variable sources found in Serpens Main. Three of the five secular variables are uncovered in this region. All are found to increase in brightness with time during the epochs observed (although EC 53 is actually periodic and is poorly fit by a linear rise in brightness, see Figure 11). The individual epochs for the other two Serpens Main sources do not appear to be influenced by the large fluctuations seen in EC 53. Indeed, EC 53 is significantly fainter than SMM 1 (see Section 5). The Serpens Main region has five independent calibrator sources, all of 
which have flat light curves (Mairs et al. 2017a), including the source SH 2-26 N whose light curve is shown in Figure 15.

Finally, an obvious extension of the JCMT Transient Survey is to use the ALMA submillimeter array to search for variations in the submillimeter morphology and brightness of deeply embedded protostars (see, e.g., Hunter et al. 2017). ALMA's images with high spatial resolution will yield important information on changes to the physical and chemical conditions within both the inner disk region and the jet/outflow emanating from these sources. Furthermore, the ALMA archive already contains many examples where protostars have been observed multiple times in the same wavelength band, with at least one continuum window and with a similar spatial resolution. The required continuum observations for these bright targets are extremely short, even to reach signal-to-noise ratios of greater than 300, and thus most of the archived measurement sets have the necessary sensitivity. The ability to achieve a precise relative calibration across multiple epochs of individual targets, however, is complicated both by variations in antenna configuration (and source rotation on the sky) and by the stability of known calibrator sources. These complications may be minimized with dedicated observations obtained with similar configurations and in the same bandpass. A first epoch of a pilot survey has been obtained in Cycle 3 (PI D. Johnstone) and is now being used to investigate best practices for data reduction and analysis to optimize the precision of the flux calibration.

\section{Summary}

The JCMT Transient Survey (Herczeg et al. 2017) is halfway through its three-year monthly monitoring of eight nearby starforming regions at submillimeter wavelengths. In this paper we have analyzed the first 18 months of Transient Survey data to uncover initial statistics on the rate of variability among protostars and disk sources. We find that only one protostar, EC 53 in Serpens Main (Yoo et al. 2017), shows large $>10 \%$ variations in its submillimeter brightness over time and note that these variations are due to an 18 month periodicity. Two additional protostars show potential stochastic variations, each having a single epoch with a strong statistical outlier measurement. Additional observations are needed to determine the significance of these rare events. We further find that five of the 150 brightest submillimeter peaks are well fit by a linear variation in brightness. All five of these sources are associated with known protostars, representing $10 \%$ of the protostellar sample within the 150 bright submillimeter peaks. Three of these secular variables are also found to be varying by Mairs et al. (2017b) through their independent analysis of submillimeter brightness changes across two to four years. None of the 100 bright peaks unassociated with protostars is found to vary. Finally, analysis of a toy model for the underlying distribution of secular brightness variations within the protostellar sample reveals that a Gaussian distribution of fractional brightness change per year of $\sigma_{S_{t}}=0.005$ would be unobservable at present, while $\sigma_{S_{t}}=0.02$ is ruled out.

The authors thank both the anonymous referee and Neal Evans for suggestions that significantly enhanced the readability of this paper. The authors gratefully acknowledge the hospitality of the Kavli Institute at Peking University where the majority of the analysis and paper writing was conducted while D.J. was a Visiting Scholar. D.J. is supported by the National
Research Council of Canada and by an NSERC Discovery Grant. S.M. was partially supported by the Natural Sciences and Engineering Research Council (NSERC) of Canada graduate scholarship program. G.H. is supported by general grant 11473005 awarded by the National Science Foundation of China. J.E.L. is supported by the Basic Science Research Program through the National Research Foundation of Korea (grant No. NRF-2015R1A2A2A01004769) and the Korea Astronomy and Space Science Institute under the R\&D program supervised by the Ministry of Science, ICT and Future Planning. M.K. is supported by Basic Science Research Program through the National Research Foundation of Korea (NRF) funded by the Ministry of Science, ICT \& Future Planning (No. NRF2015R1C1A1A01052160).

The authors wish to recognize and acknowledge the very significant cultural role and reverence that the summit of Maunakea has always had within the indigenous Hawaiian community. We are most fortunate to have the opportunity to conduct observations from this mountain.

The JCMT is operated by the East Asian Observatory on behalf of The National Astronomical Observatory of Japan, Academia Sinica Institute of Astronomy and Astrophysics, the Korea Astronomy and Space Science Institute, the National Astronomical Observatories of China and the Chinese Academy of Sciences (Grant No. XDB09000000), with additional funding support from the Science and Technology Facilities Council of the United Kingdom and participating universities in the United Kingdom and Canada. The identification number for the JCMT Transient Survey under which the SCUBA-2 data used in this paper can be found is M16AL001.

The authors thank the JCMT staff for their support of the GBS team in data collection and reduction efforts. The Starlink software (Currie et al. 2014) is supported by the East Asian Observatory.

This research has made use of NASA's Astrophysics Data System and the facilities of the Canadian Astronomy Data Centre operated by the National Research Council of Canada with the support of the Canadian Space Agency. This research used the services of the Canadian Advanced Network for Astronomy Research (CANFAR), which in turn is supported by CANARIE, Compute Canada, University of Victoria, the National Research Council of Canada, and the Canadian Space Agency.

Facility: JCMT (SCUBA-2) (Holland et al. 2013).

Software: Starlink (Currie et al. 2014), SMURF (Jenness et al. 2013), CUPID (Berry et al. 2013), Astropy (Astropy Collaboration et al. 2013), APLpy (Robitaille \& Bressert 2012), Matplotlib (Hunter 2007).

\section{ORCID iDs}

Doug Johnstone (ib https://orcid.org/0000-0002-6773-459X Gregory J. Herczeg (i) https://orcid.org/0000-0002-7154-6065 Steve Mairs (ib https://orcid.org/0000-0002-6956-0730 Jennifer Hatchell (1) https://orcid.org/0000-0002-4870-2760 Geoffrey C. Bower (iD https://orcid.org/0000-0003-4056-9982 Helen Kirk (iD https://orcid.org/0000-0002-5779-8549 James Lane (ib https://orcid.org/0000-0001-8472-6404 Graham S. Bell (i) https://orcid.org/0000-0003-0438-8228 Sarah Graves (iD https://orcid.org/0000-0001-9361-5781 Yuri Aikawa (iD https://orcid.org/0000-0003-3283-6884 
Huei-Ru Vivien Chen (16) https://orcid.org/0000-00029774-1846

Wen-Ping Chen (1) https://orcid.org/0000-0003-0262-272X

Miju Kang (1) https://orcid.org/0000-0002-5016-050X

Sung-Ju Kang (1) https://orcid.org/0000-0002-5004-7216

Jeong-Eun Lee (10) https://orcid.org/0000-0003-3119-2087

Oscar Morata (1) https://orcid.org/0000-0002-5908-9543

Andy Pon (다 https://orcid.org/0000-0003-4612-1812

Aleks Scholz (10) https://orcid.org/0000-0001-8993-5053

\section{References}

ALMA Partnership, Brogan, C. L., Pérez, L. M., et al. 2015, ApJL, 808, L3 Andrews, S. M., \& Williams, J. P. 2007, ApJ, 671, 1800

Armitage, P. J. 2015, arXiv:1509.06382

Astropy Collaboration, Robitaille, T. P., Tollerud, E. J., et al. 2013, A\&A, 558, A33

Audard, M., Ábrahám, P., Dunham, M. M., et al. 2014, in Protostars and Planets VI, ed. H. Beuther et al. (Tucson, AZ: Univ. of Arizona Press), 387

Bae, J., Hartmann, L., Zhu, Z., \& Nelson, R. P. 2014, ApJ, 795, 61

Baraffe, I., Elbakyan, V. G., Vorobyov, E. I., \& Chabrier, G. 2017, A\&A, 597, A19

Berry, D. S. 2015, A\&C, 10, 22

Berry, D. S., Reinhold, K., Jenness, T., \& Economou, F. 2013, CUPID: Clump Identification and Analysis Package, Astrophysics Source Code Library, ascl: 1311.007

Billot, N., Morales-Calderón, M., Stauffer, J. R., Megeath, S. T., \& Whitney, B. 2012, ApJL, 753, L35

Bower, G. C., Plambeck, R. L., Bolatto, A., et al. 2003, ApJ, 598, 1140

Carpenter, J. M., Hillenbrand, L. A., \& Skrutskie, M. F. 2001, AJ, 121, 3160

Chapin, E. L., Berry, D. S., Gibb, A. G., et al. 2013, MNRAS, 430, 2545

Cody, A. M., Hillenbrand, L. A., David, T. J., et al. 2017, ApJ, 836, 41

Connelley, M. S., \& Greene, T. P. 2010, AJ, 140, 1214

Costigan, G., Vink, J. S., Scholz, A., Ray, T., \& Testi, L. 2014, MNRAS, 440,3444

Coudé, S., Bastien, P., Kirk, H., et al. 2016, MNRAS, 457, 2139

Currie, M. J., Berry, D. S., Jenness, T., et al. 2014, in ASP Conf. Ser. 485 , Astronomical Data Analysis Software and Systems XXIII, ed. N. Manset \& P. Forshay (San Francisco, CA: ASP), 391

Da Rio, N., Tan, J. C., Covey, K. R., et al. 2016, ApJ, 818, 59

D'Angelo, C. R., \& Spruit, H. C. 2010, MNRAS, 406, 1208

Dempsey, J. T., \& Friberg, P. 2008, Proc. SPIE, 7012, $70123 Z$

Dempsey, J. T., Friberg, P., Jenness, T., et al. 2013, MNRAS, 430, 2534

Dionatos, O., Nisini, B., Codella, C., \& Giannini, T. 2010, A\&A, 523, A29

Drabek, E., Hatchell, J., Friberg, P., et al. 2012, MNRAS, 426, 23

Dunham, M. M., Allen, L. E., Evans, N. J., II, et al. 2015, ApJS, 220, 11

Enoch, M. L., Corder, S., Dunham, M. M., \& Duchêne, G. 2009a, ApJ, 707, 103

Enoch, M. L., Evans, N. J., II, Sargent, A. I., \& Glenn, J. 2009b, ApJ, 692, 973

Fischer, W. J., \& Hillenbrand, L. 2017, ATel, 9969

Fischer, W. J., Megeath, S. T., Furlan, E., et al. 2017, ApJ, 840, 69

Forbrich, J., Reid, M. J., Menten, K. M., et al. 2017, ApJ, 844, 109

Frimann, S., Jørgensen, J. K., Dunham, M. M., et al. 2017, A\&A, 602, A120

Furlan, E., Megeath, S. T., Osorio, M., et al. 2014, ApJ, 786, 26

Furlan, E., Fischer, W. J., Ali, B., et al. 2016, ApJS, 224, 5

Gibb, A. G., Jenness, T., \& Economou, F. 2013, in Starlink User Note 265, PICARD-A PIpeline for Combining and Analyzing Reduced Data (Hilo, HI: Joint Astronomy Centre), 265

Goicoechea, J. R., Cernicharo, J., Karska, A., et al. 2012, A\&A, 548, A77

Harsono, D., Bruderer, S., \& van Dishoeck, E. F. 2015, A\&A, 582, A41

Hartmann, L., Cassen, P., \& Kenyon, S. J. 1997, ApJ, 475, 770

Hartmann, L., Herczeg, G., \& Calvet, N. 2016, ARA\&A, 54, 135
Haschick, A. D., Moran, J. M., Rodriguez, L. F., \& Ho, P. T. P. 1983, ApJ, 265,281

Herczeg, G. J., Johnstone, D., Mairs, S., et al. 2017, ApJ, 849, 43

Hillenbrand, L. A. 1997, AJ, 113, 1733

Hodapp, K. W., Chini, R., Watermann, R., \& Lemke, R. 2012, ApJ, 744, 56 Holland, W. S., Bintley, D., Chapin, E. L., et al. 2013, MNRAS, 430, 2513

Hosokawa, T., Offner, S. S. R., \& Krumholz, M. R. 2011, ApJ, 738, 140

Hull, C. L. H., Girart, J. M., Kristensen, L. E., et al. 2016, ApJL, 823, L27

Hunter, J. D. 2007, CSE, 9, 90

Hunter, T. R., Brogan, C. L., MacLeod, G., et al. 2017, ApJL, 837, L29

Jenness, T., Chapin, E. L., Berry, D. S., et al. 2013, SMURF: SubMillimeter User Reduction Facility, Astrophysics Source Code Library, ascl:1310.007

Johnstone, D., \& Bally, J. 1999, ApJL, 510, L49

Johnstone, D., Hendricks, B., Herczeg, G. J., \& Bruderer, S. 2013, ApJ, 765,133

Jørgensen, J. K., Johnstone, D., Kirk, H., et al. 2008, ApJ, 683, 822

Kang, M., Lee, J.-E., Choi, M., et al. 2013, ApJS, 209, 25

Kim, H. J., Evans, N. J., II, Dunham, M. M., Lee, J.-E., \& Pontoppidan, K. M. 2012, ApJ, 758, 38

Kirk, H., Johnstone, D., Di Francesco, J., et al. 2016, ApJ, 821, 98

Lee, K. I., Fernández-López, M., Storm, S., et al. 2014, ApJ, 797, 76

Liu, H. B., Dunham, M. M., Pascucci, I., et al. 2017, arXiv:1710.08686

Mairs, S., Johnstone, D., Kirk, H., et al. 2015, MNRAS, 454, 2557

Mairs, S., Johnstone, D., Kirk, H., et al. 2016, MNRAS, 461, 4022

Mairs, S., Lane, J., Johnstone, D., et al. 2017a, ApJ, 843, 55

Mairs, S., Johnstone, D., Kirk, H., et al. 2017b, arXiv:1710.03761

Manara, C. F., Robberto, M., Da Rio, N., et al. 2012, ApJ, 755, 154

Manara, C. F., Testi, L., Natta, A., \& Alcalá, J. M. 2015, A\&A, 579, A66

Manoj, P., Green, J. D., Megeath, S. T., et al. 2016, ApJ, 831, 69

Maury, A. J., André, P., Men'shchikov, A., Könyves, V., \& Bontemps, S. 2011, A\&A, 535, A77

Megeath, S. T., Gutermuth, R., Muzerolle, J., et al. 2012, AJ, 144, 192

Morales-Calderón, M., Stauffer, J. R., Hillenbrand, L. A., et al. 2011, ApJ, 733,50

Mottram, J. C., van Dishoeck, E. F., Kristensen, L. E., et al. 2017, A\&A, 600, A99

Pérez, L. M., Carpenter, J. M., Andrews, S. M., et al. 2016, Sci, 353, 1519

Press, W. H., Flannery, B. P., Teukolsky, S. A., \& Vetterling, W. T. 1989, Numerical Recipes in C. The Art of Scientific Computing

Rigliaco, E., Wilking, B., Meyer, M. R., et al. 2016, A\&A, 588, A123

Robitaille, T., \& Bressert, E. 2012, APLpy: Astronomical Plotting Library in Python, Astrophysics Source Code Library, ascl:1208.017

Romanova, M. M., Ustyugova, G. V., Koldoba, A. V., \& Lovelace, R. V. E. 2012, MNRAS, 421, 63

Sadavoy, S. I., Di Francesco, J., Bontemps, S., et al. 2010, ApJ, 710, 1247

Safron, E. J., Fischer, W. J., Megeath, S. T., et al. 2015, ApJL, 800, L5

Stutz, A. M., Tobin, J. J., Stanke, T., et al. 2013, ApJ, 767, 36

Stutzki, J., \& Guesten, R. 1990, ApJ, 356, 513

Takahashi, S., Ho, P. T. P., Tang, Y.-W., Kawabe, R., \& Saito, M. 2009, ApJ, 704, 1459

Takahashi, S., Saito, M., Ohashi, N., et al. 2008, ApJ, 688, 344

Tobin, J. J., Kratter, K. M., Persson, M. V., et al. 2016a, Natur, 538, 483

Tobin, J. J., Stutz, A. M., Manoj, P., et al. 2016b, ApJ, 831, 36

Velusamy, T., Langer, W. D., \& Thompson, T. 2014, ApJ, 783, 6

Vorobyov, E. I., \& Basu, S. 2010, ApJ, 719, 1896

Ward-Thompson, D., Di Francesco, J., Hatchell, J., et al. 2007, PASP, 119, 855

Winston, E., Megeath, S. T., Wolk, S. J., et al. 2007, ApJ, 669, 493

Yoo, H., Lee, J.-E., Mairs, S., et al. 2017, ApJ, 849, 69

Yu, K. C., Billawala, Y., Smith, M. D., Bally, J., \& Butner, H. M. 2000, AJ, 120, 1974

Zhang, M., Fang, M., Wang, H., et al. 2015, ApJS, 219, 21

Zhu, Z., Hartmann, L., Gammie, C., \& McKinney, J. C. 2009, ApJ, 701, 620 